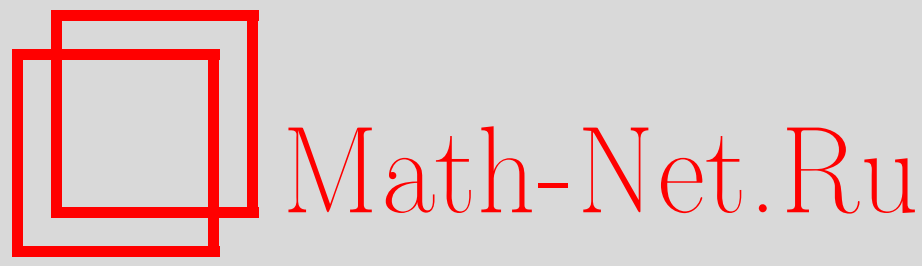

В. В. Жаринов, Дифференциально-разностный бикомплекс, ТМФ, 2010, том 165, номер 2, 195-216

DOI: https://doi.org/10.4213/tmf6571

Использование Общероссийского математического портала Math-Net.Ru подразумевает, что вы прочитали и согласны с пользовательским соглашением http: //www.mathnet.ru/rus/agreement

Параметры загрузки:

IP: 52.6 .47 .48

26 апреля 2023 г., 12:45:31

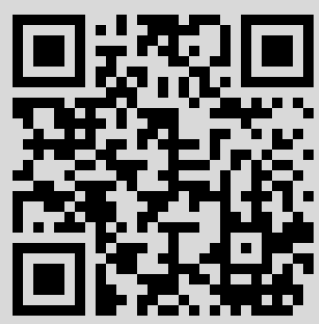




\title{
ДИФФЕРЕНЦИАЛЬНО-РАЗНОСТНЫЙ БИКОМПЛЕКС
}

\begin{abstract}
Развита техника разностных джетов на многомерной целочисленной решетке. На этой основе построен дифференциально-разностный бикомплекс решетки в классе функций локально-конечного порядка и доказана его ацикличность.
\end{abstract}

Ключевые слова: целочисленная решетка, разностные джеты, дифференциально-разностный бикомплекс.

Как хорошо известно, в теоретических и прикладных исследованиях широко используется вариационное исчисление на евклидовых пространствах, основу которого составляет вариационный комплекс (см., например, [1]), в свою очередь являющийся частью фундаментального объекта - вариационного бикомплекса (см., например, [2]-[4]). С другой стороны, в связи с бурным ростом возможностей вычислительной техники все большую популярность приобретают решеточные модели в теоретической и математической физике и разностные уравнения в практических физических и инженерных задачах, так что весьма актуальным представляется развитие вариационной техники на решетках. В рамках такой программы в настоящей работе построен дифференциально-разностный бикомплекс на многомерной целочисленной решетке в классе функций локально-конечного порядка и доказано, что он ациклический, т. е. все его строки и столбцы точные. Для этого введено понятие разностного джета и развит соответствующий аппарат. В п. 2.5 обсуждается зависимость вида дифференциально-разностного бикомплекса от выбора класса функций на пространстве разностных джетов.

Будем использовать стандартные обозначения: $\mathbb{R}$ - поле вещественных чисел, $\mathbb{Z}=\{0, \pm 1, \pm 2, \ldots\}$ - множество целых чисел, $\mathbb{Z}_{+}=\{0,1,2, \ldots\}$ - множество неотрицательных целых чисел, $\mathbb{N}=\{1,2, \ldots\}$ - множество натуральных чисел.

\section{1. РАССЛОЕНИЕ ДЖЕТОВ НАД РЕШЕТКОЙ}

1.1. Решетка. Пусть $D \in \mathbb{N}, \mathbb{D}=\{1,2, \ldots, D\}$.

Решетка (точнее, $D$-мерная целочисленная решетка) есть множество $\mathbb{L}=\mathbb{Z}^{D}$. На множестве $\mathbb{L}$ определены две согласованные алгебраические структуры: абелевой

* Математический институт им. В. А. Стеклова РАН, Москва, Россия. E-mail: zharinov@mi.ras.ru 
группы по сложению и $\mathbb{Z}$-модуля. Как $\mathbb{Z}$-модуль, $\mathbb{L}$ имеет базис $\left\{e_{1}, \ldots, e_{D}\right\}$, где

$$
e_{1}=(1,0, \ldots, 0), \ldots, e_{D}=(0, \ldots, 0,1) \text {. }
$$

Для $i=\left(i^{1}, \ldots, i^{D}\right)=\sum_{\mu \in \mathbb{D}} i^{\mu} e_{\mu} \in \mathbb{L}$ полагаем $|i|=\sum_{\mu \in \mathbb{D}}\left|i^{\mu}\right| \in \mathbb{Z}_{+}$, а для $p \in \mathbb{Z}_{+}$ полагаем $\mathbb{B}_{p}=\{i \in \mathbb{L}|| i \mid \leqslant p\}$.

На решетке (множестве) $\mathbb{L}$ определено действие группы $\mathbb{L}$,

$$
T: \mathbb{L} \rightarrow \operatorname{Aut}(\mathbb{L}), \quad i \mapsto T_{i}: \mathbb{L} \rightarrow \mathbb{L}, \quad k \mapsto T_{i} k=k+i .
$$

Пусть $\mathcal{A}(\mathbb{L})=\operatorname{Map}(\mathbb{L} ; \mathbb{R})$ - алгебра всех функций на решетке $\mathbb{L}$. Группа $\mathbb{L}$ действует на алгебре $\mathcal{A}(\mathbb{L})$ стандартным образом:

$$
T: \mathbb{L} \rightarrow \operatorname{Aut}(\mathcal{A}(\mathbb{L})), \quad i \mapsto T_{i}: \mathcal{A}(\mathbb{L}) \rightarrow \mathcal{A}(\mathbb{L}), \quad \varphi \mapsto T_{i} \varphi=\varphi \circ T_{i},
$$

так что $\left(T_{i} \varphi\right)(k)=\varphi\left(T_{i} k\right)=\varphi(k+i)$ для всех $k \in \mathbb{L}$.

1.2. Векторное расслоение над решеткой. Пусть $V$ - линейное пространство. Будем рассматривать прямое произведение $J=\mathbb{L} \times V$ как векторное расслоение над решеткой $\mathbb{L}$ с типичным слоем $V$ и проекцией на первый сомножитель:

$$
\pi: J=\mathbb{L} \times V \rightarrow \mathbb{L}, \quad(k, u) \mapsto \pi(k, u)=k .
$$

$\mathcal{A}(\mathbb{L})$-модуль всех сечений расслоения $\pi$ есть $\mathcal{M}(\mathbb{L} ; V)=\operatorname{Map}(\mathbb{L} ; V)-$ множество всех отображений из $\mathbb{L}$ в $V$. Считая, что группа $\mathbb{L}$ действует на слоях тривиально, получим ее действие на расслоении $\pi$,

$$
T: \mathbb{L} \rightarrow \operatorname{Aut}(J), \quad i \mapsto T_{i}: J \rightarrow J, \quad(k, u) \mapsto T_{i}(k, u)=\left(T_{i} k, u\right) .
$$

Индуцированное действие на сечениях имеет вид

$$
T: \mathbb{L} \rightarrow \operatorname{Aut}(\mathcal{M}(\mathbb{L} ; V)), \quad i \mapsto T_{i}: \mathcal{M}(\mathbb{L} ; V) \rightarrow \mathcal{M}(\mathbb{L} ; V), \quad \varphi \mapsto T_{i} \varphi,
$$

где $T_{i} \varphi=\varphi \circ T_{i}$, т. е. $\left(T_{i} \varphi\right)(k)=\varphi(k+i)$ для всех $i, k \in \mathbb{L}, \varphi \in \mathcal{M}(\mathbb{L} ; V)$.

1.3. Расслоение разностных джетов над решеткой. Пусть $\pi: J=\mathbb{L} \times V \rightarrow$ $\mathbb{L}$ - векторное расслоение над $\mathbb{L}$. Будем говорить, что сечения $\varphi, \psi \in \mathcal{M}(\mathbb{L} ; V)$ касаются над точкой $k \in \mathbb{L}$ с порядком $p \in \mathbb{Z}_{+}$, если $\varphi(k+i)=\psi(k+i)$ для всех $i \in \mathbb{B}_{p}$. Будем также говорить, что сечения $\varphi, \psi \in \mathcal{M}(\mathbb{L} ; V)$ касаются над точкой $k \in \mathbb{L}$ с порядком $\infty$, если $\varphi(k+i)=\psi(k+i)$ для всех $i \in \mathbb{B}_{\infty}=\mathbb{L}$, т. е. если сечения $\varphi$ и $\psi$ совпадают.

При таком определении касания векторное расслоение разностных джетов порядка $р \in \mathbb{Z}_{+}$над решеткой $\mathbb{L}$ есть

$$
\boldsymbol{\pi}_{p}: \mathbf{J}_{p}=\mathbb{L} \times \mathbf{V}_{p} \rightarrow \mathbb{L}, \quad(k, \mathbf{u}) \mapsto \boldsymbol{\pi}_{p}(k, \mathbf{u})=k,
$$

где типичный слой

$$
\mathbf{V}_{p}=\left\{\mathbf{u}=\mathbf{u}_{p}=\left(u_{l}\right) \mid l \in \mathbb{B}_{p}, u_{l} \in V\right\}=V^{\mathbb{B}_{p}} .
$$

Будем считать, что $\mathbf{V}_{0}=V, \mathbf{J}_{0}=J, \boldsymbol{\pi}_{0}=\pi$. 
$\mathcal{A}(\mathbb{L})$-модуль всех сечений расслоения $\boldsymbol{\pi}_{p}$ есть множество

$$
\mathcal{M}\left(\mathbb{L} ; \mathbf{V}_{p}\right)=\operatorname{Map}\left(\mathbb{L} ; \mathbf{V}_{p}\right)=\left\{\phi=\phi_{p}=\left(\varphi_{l}\right) \mid l \in \mathbb{B}_{p}, \varphi_{l} \in \mathcal{M}(\mathbb{L} ; V)\right\}
$$

Определено отображение

$$
\boldsymbol{j}_{p}: \mathcal{M}(\mathbb{L} ; V) \rightarrow \mathcal{M}\left(\mathbb{L} ; \mathbf{V}_{p}\right), \quad \varphi \mapsto \boldsymbol{j}_{p} \varphi, \quad\left(\boldsymbol{j}_{p} \varphi\right)_{l}=T_{l} \varphi, \quad l \in \mathbb{B}_{p}
$$

Сечения $\varphi, \psi \in \mathcal{M}(\mathbb{L} ; V)$ касаются над точкой $k$ с порядком $p$ тогда и только тогда, когда $\left(\boldsymbol{j}_{p} \varphi\right)(k)=\left(\boldsymbol{j}_{p} \psi\right)(k)$, т. е. когда их $p$-джеты в точке $k$ совпадают.

Пусть $p, q \in \mathbb{Z}_{+}, p \geqslant q$. Естественное вложение $\mathbb{B}_{q} \subset \mathbb{B}_{p}$ индуцирует проекцию

$$
\boldsymbol{\pi}_{p q}: \mathbf{J}_{p} \rightarrow \mathbf{J}_{q}, \quad \boldsymbol{\pi}_{q} \circ \boldsymbol{\pi}_{p q}=\boldsymbol{\pi}_{p}
$$

так что определен проективный предел - векторное расслоение разностных джетов (бесконечного порядка)

$$
\boldsymbol{\pi}=\boldsymbol{\pi}_{\infty}: \mathbf{J}=\mathbf{J}_{\infty}=\mathbb{L} \times \mathbf{V} \rightarrow \mathbb{L}, \quad(k, \mathbf{u}) \mapsto \boldsymbol{\pi}(k, \mathbf{u})=k,
$$

где типичный слой

$$
\mathbf{V}=\mathbf{V}_{\infty}=\operatorname{limproj}_{p \rightarrow \infty} \mathbf{V}_{p}=\left\{\mathbf{u}=\mathbf{u}_{\infty}=\left(u_{l}\right) \mid l \in \mathbb{L}, u_{l} \in V\right\}=V_{\mathbb{L}}
$$

$\mathcal{A}(\mathbb{L})$-модуль всех сечений расслоения $\boldsymbol{\pi}$ есть

$$
\mathcal{M}(\mathbb{L} ; \mathbf{V})=\operatorname{Map}(\mathbb{L} ; \mathbf{V})=\left\{\boldsymbol{\phi}=\phi_{\infty}=\left(\varphi_{l}\right) \mid l \in \mathbb{L}, \varphi_{l} \in \mathcal{M}(\mathbb{L} ; V)\right\} .
$$

По построению $\mathbf{V}=V_{\mathbb{L}}=\operatorname{Map}(\mathbb{L} ; V)=\mathcal{M}(\mathbb{L} ; V)$. Действие группы $\mathbb{L}$ на типичном слое $\mathbf{V}$ определяется согласно ее действию на $\mathcal{M}(\mathbb{L} ; V)$ :

$$
T: \mathbb{L} \rightarrow \operatorname{Aut}(\mathbf{V}), \quad i \mapsto T_{i}: \mathbf{V} \rightarrow \mathbf{V}, \quad \mathbf{u} \mapsto T_{i} \mathbf{u}, \quad\left(T_{i} \mathbf{u}\right)_{l}=u_{l+i}, \quad l \in \mathbb{L}
$$

Соответственно, действие группы $\mathbb{L}$ на расслоении $\boldsymbol{\pi}$ имеет вид

$$
T: \mathbb{L} \rightarrow \operatorname{Aut}(\mathbf{J}), \quad i \mapsto T_{i}: \mathbf{J} \rightarrow \mathbf{J}, \quad(k, \mathbf{u}) \mapsto T_{i}(k, \mathbf{u})=\left(T_{i} k, T_{i} \mathbf{u}\right),
$$

а на его сечениях -

$$
T: \mathbb{L} \rightarrow \operatorname{Aut}(\mathcal{M}(\mathbb{L} ; \mathbf{V})), \quad i \mapsto T_{i}: \mathcal{M}(\mathbb{L} ; \mathbf{V}) \rightarrow \mathcal{M}(\mathbb{L} ; \mathbf{V}), \quad \phi \mapsto T_{i} \phi
$$

где $T_{i} \phi=T_{-i} \circ \phi \circ T_{i}$, так что $\left(T_{i} \phi\right)_{l}(k)=\varphi_{l-i}(k+i), i, l, k \in \mathbb{L}$.

Определено отображение

$$
\boldsymbol{j}: \mathcal{M}(\mathbb{L} ; V) \rightarrow \mathcal{M}(\mathbb{L} ; \mathbf{V}), \quad \varphi \mapsto \boldsymbol{j} \varphi, \quad(\boldsymbol{j} \varphi)_{l}=T_{l} \varphi, \quad l \in \mathbb{L} .
$$

Сечения $\varphi, \psi \in \mathcal{M}(\mathbb{L} ; V)$ касаются над точкой $k$ с порядком $\infty$ тогда и только тогда, когда $(\boldsymbol{j} \varphi)(k)=(\boldsymbol{j} \psi)(k)$, т. е. когда их $\infty$-джеты в точке $k$ совпадают.

Сечение $\phi \in \mathcal{M}(\mathbb{L} ; \mathbf{V})$ называется инвариантным относительно сдвигов, если $T_{i} \phi=\phi$ для всех $i \in \mathbb{L}$. Линейное пространство всех таких сечений обозначим через $\mathcal{M}_{T}(\mathbb{L} ; \mathbf{V})$. 
ПрЕДЛОЖЕНИЕ 1. Имеют место следующие утверждения:

1. $T_{i} \circ \boldsymbol{j}=\boldsymbol{j}$ для всех $i \in \mathbb{L}$.

2. $\boldsymbol{j}: \mathcal{M}(\mathbb{L} ; V) \simeq \mathcal{M}_{T}(\mathbb{L} ; \mathbf{V})$.

3. Для каждой точки $(k, \mathbf{u}) \in \mathbf{J}$ существует одно и только одно сечение $\varphi \in$ $\mathcal{M}(\mathbb{L} ; V)$ maкое, что $(\boldsymbol{j}(\varphi))(k)=\mathbf{u}$.

ДокаЗАтельство. Действительно, для $i \in \mathbb{L}, \varphi \in \mathcal{M}(\mathbb{L} ; V), l, k \in \mathbb{L}$, имеем

$$
\left(T_{i} \boldsymbol{j} \varphi\right)_{l}(k)=(\boldsymbol{j} \varphi)_{l-i}(k+i)=\varphi(k+i+l-i)=\varphi(k+l)=(\boldsymbol{j} \varphi)_{l}(k) .
$$

Далее, пусть $\phi \in \mathcal{M}(\mathbb{L} ; \mathbf{V})$ и $T_{i} \phi=\phi, i \in \mathbb{L}$, т. е. $\varphi_{l-i}(k+i)=\varphi_{l}(k)$ для всех $i, l, k \in \mathbb{L}$. В частности, при $i=l$ имеем $\varphi_{0}(k+l)=\varphi_{l}(k)$, т. е. $\varphi_{l}=T_{l} \varphi_{0}$ для всех $l \in \mathbb{L}$, и, значит, $\boldsymbol{\phi}=\boldsymbol{j} \varphi_{0}$. Наконец, пусть $(k, \mathbf{u}) \in \mathbf{J}$. Определим сечение $\varphi \in \mathcal{M}(\mathbb{L} ; V)$ равенством $\varphi(i)=u_{i-k}$ для всех $i \in \mathbb{L}$. Тогда $(\boldsymbol{j} \varphi)_{l}(k)=\varphi(k+l)=u_{k+l-k}=u_{l}$, что и требовалось доказать. Единственность очевидна.

1.4. Функции на пространстве разностных джетов. Пусть $\pi: J=\mathbb{L} \times$ $V \rightarrow \mathbb{L}$ - векторное расслоение, $W$ - линейное пространство.

Для каждого $p \in \mathbb{Z}_{+}$обозначим через $\mathcal{A}\left(\mathbf{V}_{p}\right)=\mathcal{C}^{\infty}\left(\mathbf{V}_{p} ; \mathbb{R}\right)$ алгебру всех гладких функций на пространстве $\mathbf{V}_{p}$ с естественной локально-выпуклой топологией, а через

$$
\mathcal{A}(\mathbf{V})=\mathcal{C}^{\infty}(\mathbf{V} ; \mathbb{R})=\operatorname{limind}_{p \rightarrow \infty} \mathcal{A}\left(\mathbf{V}_{p}\right)
$$

- индуктивный предел таких алгебр относительно естественного вложения. По построению алгебра $\mathcal{A}(\mathbf{V})$ состоит из всех функций $f(\mathbf{u})$ на пространстве $\mathbf{V}$, каждая из которых зависит лишь от конечного числа переменных $u_{l}$ и гладкая по этим переменным. Далее, обозначим через $\mathcal{A}(\mathbf{J})=\mathcal{A}(\mathbb{L}) \otimes \mathcal{A}(\mathbf{V})$ алгебру всех функций $f(k, \mathbf{u})$ на пространстве $\mathbf{J}$ таких, что при каждом $k \in \mathbb{L}$ функция $g_{k}(\mathbf{u}) \in \mathcal{A}(\mathbf{V})$. Заметим, что имеется естественное вложение $\mathcal{A}(\mathbf{V}) \subset \mathcal{A}(\mathbf{J}), f(\mathbf{u}) \mapsto f(k, \mathbf{u})=f(\mathbf{u})$. Наконец, обозначим через $\mathcal{M}(\mathbf{J} ; W)=\mathcal{A}(\mathbf{J}) \otimes W \mathcal{A}(\mathbf{J})$-модуль всех отображений $f(k, \mathbf{u})$ из пространства $\mathbf{J}$ в $W$ таких, что при каждом $k \in \mathbb{L}$ отображение $g_{k}(\mathbf{u}) \in \mathcal{M}(\mathbf{V} ; W)=$ $\mathcal{A}(\mathbf{V}) \otimes W$.

Имеется альтернативный выбор алгебры гладких функций на пространстве разностных джетов. Именно, для каждого $p \in \mathbb{Z}_{+}$обозначим через $\mathcal{A}\left(\mathbf{J}_{p}\right)=\mathcal{A}(\mathbb{L}) \otimes$ $\mathcal{A}\left(\mathbf{V}_{p}\right)$ алгебру всех функиий разностного порядка $p$, а через

$$
\mathcal{A}_{\text {fin }}(\mathbf{J})=\operatorname{limind}_{p \rightarrow \infty} \mathcal{A}\left(\mathbf{J}_{p}\right)
$$

- алгебру всех функиий на пространстве $\mathbf{J}$ конечного разностного порядка. Имеется по-прежнему естественное вложение $\mathcal{A}(\mathbf{V}) \subset \mathcal{A}_{\text {fin }}(\mathbf{J})$.

Очевидно, $\mathcal{A}_{\text {fin }}(\mathbf{J}) \subsetneq \mathcal{A}(\mathbf{J})$. Более того, по построению каждая функция $f(k, \mathbf{u}) \in$ $\mathcal{A}_{\mathrm{fin}}(\mathbf{J})$ имеет глобальный разностный порядок $p=p(f)$, так что $\partial_{u_{l}} f(k, \mathbf{u})=0$ для всех $k \in \mathbb{L}, \mathbf{u} \in \mathbf{V}$ и $|l|>p$, тогда как каждая функция $f(k, \mathbf{u}) \in \mathcal{A}(\mathbf{J})$ обязана лишь для каждого $k \in \mathbb{L}$ иметь локалъный разностный порядок $p=p(f, k)$, так что здесь $\partial_{u_{l}} f(k, \mathbf{u})=0$ для всех $\mathbf{u} \in \mathbf{V}$ и $|l|>p$.

Ниже мъ ограничиваемся алгеброй $\mathcal{A}(\mathbf{J})$. Алгебра $\mathcal{A}_{\text {fin }}(\mathbf{J})$ требует отдельного рассмотрения. 
Отображения $f \in \mathcal{M}(\mathbf{J} ; W)$ суть сечения векторного расслоения

$$
\mathbf{J} \times W \rightarrow \mathbf{J}, \quad(k, \mathbf{u}, w) \mapsto(k, \mathbf{u})
$$

Считая, что сдвиги действуют на слоях тривиально, получим действие группы $\mathbb{L}$ на этом расслоении

$$
\begin{aligned}
T: \mathbb{L} \rightarrow \operatorname{Aut}(\mathbf{J} \times W), \quad & \mapsto \mapsto T_{i}: \mathbf{J} \times W \\
(k, \mathbf{u}, w) & \mapsto \mathbf{J} \times W, \\
& \mapsto(k, \mathbf{u}, w)=\left(T_{i} k, T_{i} \mathbf{u}, w\right) .
\end{aligned}
$$

На сечениях соответственно имеем

$$
T: \mathbb{L} \rightarrow \operatorname{Aut}(\mathcal{M}(\mathbf{J} ; W)), \quad i \mapsto T_{i}: \mathcal{M}(\mathbf{J} ; W) \rightarrow \mathcal{M}(\mathbf{J} ; W), \quad f \mapsto T_{i} f
$$

где $T_{i} f=f \circ T_{i}$, так что $\left(T_{i} f\right)(k, \mathbf{u})=f\left(T_{i} k, T_{i} \mathbf{u}\right)$.

Для каждого сечения $\phi \in \mathcal{M}(\mathbb{L} ; \mathbf{V})$ определена подстановка

$$
\left.\right|_{\phi}: \mathcal{M}(\mathbf{J} ; W) \rightarrow \mathcal{M}(\mathbb{L} ; W),\left.\quad f \mapsto f\right|_{\phi},\left.\quad f\right|_{\phi}(k)=f(k, \phi(k)) .
$$

ПРЕДЛОЖЕНИЕ 2 (ПРАВИЛО ПОЛНОГО СДВИГА). Для $\phi \in \mathcal{M}(\mathbb{L} ; \mathbf{V})$ равенство $\left.\left(T_{i} f\right)\right|_{\phi}=T_{i}\left(\left.f\right|_{\phi}\right)$ справедливо для всех $i \in \mathbb{L} u f \in \mathcal{M}$ тогда и только тогда, когда $\phi=\boldsymbol{j}(\varphi)$, где сечение $\varphi=\varphi_{0} \in \mathcal{M}(\mathbb{L} ; V)$.

ДокАЗАТЕЛЬСТво. По построению

$$
\left.\left(T_{i} f\right)\right|_{\phi}(k)=f\left(T_{i} k, T_{i} \phi(k)\right), \quad T_{i}\left(\left.f\right|_{\phi}\right)(k)=f\left(T_{i} k, \phi(k)\right),
$$

так что доказываемое утверждение следует из предложения 1.

Функция $f \in \mathcal{A}(\mathbf{J})$ называется инвариантной относительно сдвигов, если $T_{i} f=f$ для всех $i \in \mathbb{L}$. Подалгебру всех таких функций обозначим через $\mathcal{A}_{T}(\mathbf{J})$.

ПрЕДЛОЖЕНИЕ 3. Имеет место изоморфизм

$$
\mathcal{A}_{T}(\mathbf{J}) \simeq \mathcal{A}(\mathbf{V}), \quad f \mapsto g, \quad g(\mathbf{u})=f(0, \mathbf{u}), \quad f(k, \mathbf{u})=g\left(T_{-k} \mathbf{u}\right) .
$$

ДокАЗАтЕЛЬСтво. Условие $T_{i} f=f$ для всех $i \in \mathbb{L}$ сводится к равенству $f\left(k+i, T_{i} \mathbf{u}\right)=f(k, \mathbf{u})$ для всех $i, k \in \mathbb{L}, \mathbf{u} \in \mathbf{V}$, так что достаточно положить $i=-k$.

СЛЕДСТВИЕ 1. $\mathcal{A}_{T}(\mathbf{J}) \cap \mathcal{A}_{\text {fin }}(\mathbf{J})=\mathbb{R}$.

1.5. Вертикальные дифференцирования. Пусть $\mathfrak{D}(\mathcal{A}(\mathbf{V}))$ - линейное пространство всех дифференцирований алгебры $\mathcal{A}(\mathbf{V})$. Как известно, на $\mathfrak{D}(\mathcal{A}(\mathbf{V}))$ определены две согласованные алгебраические структуры: структура алгебры Ли и структура $\mathcal{A}(\mathbf{V})$-модуля. В свою очередь, линейное пространство всех дифференцирований алгебры $\mathcal{A}(\mathbf{J})$ есть $\mathfrak{D}(\mathcal{A}(\mathbf{J}))=\mathcal{A}(\mathbb{L}) \otimes_{\mathbb{R}} \mathfrak{D}(\mathcal{A}(\mathbf{V}))$. На $\mathfrak{D}(\mathcal{A}(\mathbf{J}))$ также определены две согласованные алгебраические структуры: структура алгебры Ли и структура $\mathcal{A}(\mathbf{J})$-модуля. 
ЗАмЕчаниЕ 1. Образно говоря, дифференцирования в алгебре $\mathcal{A}(\mathbf{J})$ производятся по гладким переменным $\mathbf{u}$, целочисленные переменные $k$ здесь играют роль параметров. С точки зрения расслоения $\boldsymbol{\pi}$ дифференцирования в алгебре $\mathcal{A}(\mathbf{J})$ являются вертикальными, тогда как сдвиги в алгебре $\mathcal{A}(\mathbf{J})$ суть подъемы сдвигов из базы $\mathbb{L}$ и в силу этого называются горизонтальными. Отметим также, что вертикальные дифференцирования удовлетворяют правилу Лейбница $X(f g)=(X f) g+f(X g)$ для всех $X \in \mathfrak{D}(\mathcal{A}(\mathbf{J})), f, g \in \mathcal{A}(\mathbf{J})$, а горизонтальные сдвиги являются морфизмами алгебры $\mathcal{A}(\mathbf{J}), T_{i}(f g)=\left(T_{i} f\right)\left(T_{i} g\right)$ для всех $i \in \mathbb{L}, f, g \in \mathcal{A}(\mathbf{J})$. Соответственно вертикальные дифференцирования образуют алгебру Ли, а горизонтальные сдвиги абелеву группу.

Пусть $\left\{b_{\alpha} \mid \alpha \in \mathrm{A}\right\}$ - базис линейного пространства $V$, так что

$$
V \ni u=\left(u^{\alpha} \in \mathbb{R} \mid \alpha \in \mathrm{A}\right), \quad \mathbf{V} \ni \mathbf{u}=\left(u_{l}^{\alpha} \in \mathbb{R} \mid \alpha \in \mathrm{A}, l \in \mathbb{L}\right) .
$$

$\mathcal{A}(\mathbf{V})$-модуль $\mathfrak{D}(\mathcal{A}(\mathbf{V}))$ имеет в этом случае базис $\left\{\partial_{u_{l}^{\alpha}} \mid \alpha \in \mathrm{A}, l \in \mathbb{L}\right\}$, так что

$$
\mathfrak{D}(\mathcal{A}(\mathbf{V}))=\left\{X=\sum_{\alpha, l} X_{l}^{\alpha} \cdot \partial_{u_{l}^{\alpha}} \mid X_{l}^{\alpha} \in \mathcal{A}(\mathbf{V})\right\} .
$$

Дуальный модуль $\mathfrak{D}^{*}(\mathcal{A}(\mathbf{V}))=\operatorname{Hom}_{\mathcal{A}(\mathbf{V})}(\mathfrak{D}(\mathcal{A}(\mathbf{V})) ; \mathcal{A}(\mathbf{V}))$ имеет дуальный базис

$$
\left\{d_{V} u_{l}^{\alpha} \mid \alpha \in \mathrm{A}, l \in \mathbb{L}\right\}, \quad\left\langle d_{V} u_{l}^{\alpha}, \partial_{u_{j}^{\beta}}\right\rangle=\delta_{\beta}^{\alpha} \delta_{l}^{j}, \quad \alpha, \beta \in \mathrm{A}, l, j \in \mathbb{L},
$$

так что

$$
\mathfrak{D}^{*}(\mathcal{A}(\mathbf{V}))=\left\{\omega=\sum_{\alpha, l} \omega_{\alpha}^{l} \cdot d_{V} u_{l}^{\alpha} \mid \omega_{\alpha}^{l} \in \mathcal{A}(\mathbf{V})\right\},
$$

где лишь конечное число компонент $\omega_{\alpha}^{l} \neq 0$.

Аналогичные представления справедливы для $\mathcal{A}(\mathbf{J})$-модулей $\mathfrak{D}(\mathcal{A}(\mathbf{J}))$ и $\mathfrak{D}^{*}(\mathcal{A}(\mathbf{J}))$, где компоненты $X_{l}^{\alpha}, \omega_{\alpha}^{l} \in \mathcal{A}(\mathbf{J})$.

На линейном пространстве $\mathfrak{D}(\mathcal{A}(\mathbf{J}))$ определено присоединенное действие алгебры Ли $\mathfrak{D}(\mathcal{A}(\mathbf{J}))$ :

$$
\begin{array}{r}
\mathfrak{D}(\mathcal{A}(\mathbf{J})) \rightarrow \mathfrak{g l}(\mathfrak{D}(\mathcal{A}(\mathbf{J}))), \quad X \mapsto X: \mathfrak{D}(\mathcal{A}(\mathbf{J})) \rightarrow \mathfrak{D}(\mathcal{A}(\mathbf{J})), \\
Y \mapsto X(Y)=[X, Y] .
\end{array}
$$

$\mathrm{B}$ частности, $\partial_{u_{j}^{\alpha}}\left(\partial_{u_{l}^{\beta}}\right)=0$ для всех $\alpha, \beta \in \mathrm{A}, j, l \in \mathbb{L}$.

На дуальном пространстве $\mathfrak{D}^{*}(\mathcal{A}(\mathbf{J}))$ действие алгебры Ли $\mathfrak{D}(\mathcal{A}(\mathbf{J}))$ определяется как производная Ли:

$$
\begin{aligned}
& \mathfrak{D}(\mathcal{A}(\mathbf{J})) \rightarrow \mathfrak{g l}\left(\mathfrak{D}^{*}(\mathcal{A}(\mathbf{J}))\right), \quad X \mapsto X: \mathfrak{D}^{*}(\mathcal{A}(\mathbf{J})) \rightarrow \mathfrak{D}^{*}(\mathcal{A}(\mathbf{J})), \\
& \omega \mapsto X(\omega)=[X, \omega],
\end{aligned}
$$

так что $\langle X(\omega), Y\rangle=X(\langle\omega, Y\rangle)-\langle\omega,[X, Y]\rangle$ для всех $X, Y \in \mathfrak{D}(\mathcal{A}(\mathbf{J})), \omega \in \mathfrak{D}^{*}(\mathcal{A}(\mathbf{J}))$. В частности, $\partial_{u_{j}^{\alpha}}\left(d_{V} u_{l}^{\beta}\right)=0$ для всех $\alpha, \beta \in \mathrm{A}, j, l \in \mathbb{L}$.

Действие

$$
T: \mathbb{L} \rightarrow \operatorname{Aut}(\mathfrak{D}(\mathcal{A}(\mathbf{J}))), \quad i \mapsto T_{i}: \mathfrak{D}(\mathcal{A}(\mathbf{J})) \rightarrow \mathfrak{D}(\mathcal{A}(\mathbf{J})), \quad X \mapsto T_{i}(X),
$$


определяется как подъем действия с $\mathcal{A}(\mathbf{J})$, т. е. условием

$$
T_{i}(X) \circ T_{i}=T_{i} \circ X, \quad \text { так что } \quad T_{i}(X)=T_{i} \circ X \circ T_{-i} .
$$

В частности, $T_{i}\left(\partial_{u_{l}^{\alpha}}\right)=\partial_{u_{l+i}^{\alpha}}, \alpha \in \mathrm{A}, i, l \in \mathbb{L}$.

Дуальным образом действие группы $\mathbb{L}$ на дуальном пространстве $\mathfrak{D}^{*}(\mathcal{A}(\mathbf{J}))$ задается правилом

$$
\begin{aligned}
T: \mathbb{L} \rightarrow \operatorname{Aut}\left(\mathfrak{D}^{*}(\mathcal{A}(\mathbf{J}))\right), \quad i & \mapsto T_{i}: \mathfrak{D}^{*}(\mathcal{A}(\mathbf{J})) \rightarrow \mathfrak{D}^{*}(\mathcal{A}(\mathbf{J})), \\
\omega & \mapsto T_{i}(\omega)=T_{i} \circ \omega \circ T_{-i}
\end{aligned}
$$

В частности, $T_{i}\left(d_{V} u_{l}^{\alpha}\right)=d_{V} u_{l+i}^{\alpha}, \alpha \in \mathrm{A}, i, l \in \mathbb{L}$.

Дифференцирование $X \in \mathfrak{D}(\mathcal{A}(\mathbf{J}))$ называется инвариантным относительно сдвигов, если $T_{i}(X)=X$, т. е. $T_{i} \circ X=X \circ T_{i}$ для всех $i \in \mathbb{L}$. Подалгебру Ли всех таких дифференцирований обозначим через $\mathfrak{D}_{T}(\mathcal{A}(\mathbf{J}))$.

ПрЕДЛОЖЕНИЕ 4. Имеют место следующие представления:

1) $\mathfrak{D}_{T}(\mathcal{A}(\mathbf{J}))=\left\{X=\sum_{\alpha, l}\left(T_{l} f^{\alpha}\right) \cdot \partial_{u_{l}^{\alpha}} \mid f^{\alpha} \in \mathcal{A}(\mathbf{J})\right\}$

2) $\mathfrak{D}_{T}(\mathcal{A}(\mathbf{J})) \simeq \mathfrak{D}(\mathcal{A}(\mathbf{V})), X=\sum_{\alpha, l}\left(T_{l} f^{\alpha}\right) \cdot \partial_{u_{l}^{\alpha}} \mapsto Y=\sum_{\alpha, l} g_{l}^{\alpha} \cdot \partial_{u_{l}^{\alpha}}$, где $g_{l}^{\alpha}(\mathbf{u})=f^{\alpha}\left(l, T_{l} \mathbf{u}\right), f^{\alpha}(k, \mathbf{u})=g_{k}\left(T_{-k} \mathbf{u}\right)$.

Доказательство проводится прямыми вычислениями.

1.6. Горизонтальные разностные операторы. Разностные операторы $\Delta_{\mu}=$ $T_{e_{\mu}}-T_{0}, \mu \in \mathbb{D}$, действуют во всех пространствах, где определено действие группы сдвигов $\mathbb{L}$.

ПРЕДЛОЖЕНИЕ 5. Пусть $f \in \mathcal{M}(\mathbb{L} ; W)$ и ряд $\sum f=\sum_{k \in \mathbb{L}} f(k)$ абсолютно сходится. Тогда для всех $i \in \mathbb{L}$ ряд $\sum T_{i} f=\sum_{k \in \mathbb{L}} f(k+i)$ также абсолютно сходится, причем $\sum T_{i} f=\sum f$. В частности, в этом случае $\sum \Delta_{\mu} f=0$ для всех $\mu \in \mathbb{D}$.

Доказательство проводится стандартными рассуждениями.

Введем свободный $\mathcal{A}(\mathbb{L})$-модуль

$$
\mathfrak{D}(\mathbb{L})=\left\{\zeta=\sum_{\mu} \zeta^{\mu} \cdot \Delta_{\mu} \mid \zeta^{\mu} \in \mathcal{A}(\mathbb{L})\right\} \subset \operatorname{End}_{\mathbb{R}}(\mathcal{A}(\mathbb{L}))
$$

с базисом $\left\{\Delta_{1}, \ldots, \Delta_{D}\right\}$.

Дуальный $\mathcal{A}(\mathbb{L})$-модуль $\mathfrak{D}^{*}(\mathbb{L})=\operatorname{Hom}_{\mathcal{A}(\mathbb{L})}(\mathfrak{D}(\mathbb{L}) ; \mathcal{A}(\mathbb{L}))$ имеет дуальный базис $\left\{d_{H} i^{1}, \ldots, d_{H} i^{D}\right\}$, где $\left\langle d_{H} i^{\mu}, \Delta_{\nu}\right\rangle=\delta_{\nu}^{\mu}$ для всех $\mu, \nu \in \mathbb{D}$, так что

$$
\mathfrak{D}^{*}(\mathbb{L})=\left\{\varrho=\sum_{\mu} \varrho_{\mu} \cdot d_{H} i^{\mu} \mid \varrho_{\mu} \in \mathcal{A}(\mathbb{L})\right\} .
$$

Группа $\mathbb{L}$ действует на $\mathcal{A}(\mathbb{L})$-модулях $\mathfrak{D}(\mathbb{L})$ и $\mathfrak{D}^{*}(\mathbb{L})$ покомпонентно (естественное действие группы $\mathbb{L}$ на базисные разностные операторы $\Delta_{\mu}$ тривиальное, $T_{i}\left(\Delta_{\mu}\right)=$ $\left.\Delta_{\mu}\right)$ :

$$
\begin{aligned}
& T: \mathbb{L} \rightarrow \operatorname{Aut}(\mathfrak{D}(\mathbb{L})), \quad i \mapsto T_{i}: \mathfrak{D}(\mathbb{L}) \rightarrow \mathfrak{D}(\mathbb{L}), \quad \zeta \mapsto T_{i} \zeta, \\
& T: \mathbb{L} \rightarrow \operatorname{Aut}\left(\mathfrak{D}^{*}(\mathbb{L})\right), \quad i \mapsto T_{i}: \mathfrak{D}^{*}(\mathbb{L}) \rightarrow \mathfrak{D}^{*}(\mathbb{L}), \quad \varrho \mapsto T_{i} \varrho,
\end{aligned}
$$


где

$$
\begin{array}{lll}
\zeta=\sum_{\mu} \zeta^{\mu} \cdot \Delta_{\mu} & \mapsto & T_{i} \zeta=\sum_{\mu}\left(T_{i} \zeta^{\mu}\right) \cdot \Delta_{\mu}, \\
\varrho=\sum_{\mu} \varrho_{\mu} \cdot d_{H} i^{\mu} & \mapsto & T_{i} \varrho=\sum_{\mu}\left(T_{i} \varrho_{\mu}\right) \cdot d_{H} i^{\mu} .
\end{array}
$$

\section{2. БИКОМПЛЕКС}

2.1. Вертикальный дифференциальный комплекс. Определена градуированная внешняя алгебра дифференциальных форм

$$
\Omega(\mathbf{V})=\Omega(\mathcal{A}(\mathbf{V}))=\bigoplus_{p \in \mathbb{Z}_{+}} \Omega^{p}(\mathbf{V}),
$$

где $\mathcal{A}(\mathbf{V})$-модули

$$
\Omega^{p}(\mathbf{V})=\Omega^{p}(\mathcal{A}(\mathbf{V}))=\operatorname{Hom}_{\mathcal{A}(\mathbf{V})}\left(\wedge_{\mathcal{A}(\mathbf{V})}^{p} \mathfrak{D}(\mathcal{A}(\mathbf{V})) ; \mathcal{A}(\mathbf{V})\right)=\wedge_{\mathcal{A}(\mathbf{V})}^{p} \mathfrak{D}^{*}(\mathcal{A}(\mathbf{V})) .
$$

Пусть $\left\{b_{\alpha} \mid \alpha \in \mathrm{A}\right\}$ - базис линейного пространства $V, p \in \mathbb{Z}_{+}$. Положим $\boldsymbol{\alpha}=$ $\left(\alpha_{1}, \ldots, \alpha_{p}\right) \in \mathrm{A}^{p}, \mathbf{i}=\left(i_{1}, \ldots, i_{p}\right) \in \mathbb{L}^{p}$ и будем писать для такой пары мультииндексов $|\boldsymbol{\alpha}, \mathbf{i}|=p$.

В таких обозначениях

$$
\Omega^{p}(\mathbf{V})=\left\{\chi=\frac{1}{p !} \sum_{|\boldsymbol{\alpha}, \mathbf{i}|=p} \chi_{\boldsymbol{\alpha}}^{\mathbf{i}} \cdot d_{V} u_{\mathbf{i}}^{\boldsymbol{\alpha}} \mid \chi_{\boldsymbol{\alpha}}^{\mathbf{i}} \in \mathcal{A}(\mathbf{V})\right\}
$$

где:

1) лишь конечное число компонент $\chi_{\boldsymbol{\alpha}}^{\mathbf{i}} \neq 0$,

2) $\chi_{\sigma(\boldsymbol{\alpha})}^{\sigma(\mathbf{i})}=\operatorname{sign}(\sigma) \chi_{\boldsymbol{\alpha}}^{\mathbf{i}}$ для всех перестановок $\sigma \in \Sigma_{p}$,

3) $d_{V} u_{\mathbf{i}}^{\alpha}=d_{V} u_{i_{1}}^{\alpha_{1}} \wedge \cdots \wedge d_{V} u_{i_{p}}^{\alpha_{p}}$.

По стандартным правилам дифференциальной геометрии определяются линейные отображения:

1) $\partial_{u_{i}^{\alpha}}: \Omega^{p}(\mathbf{V}) \rightarrow \Omega^{p}(\mathbf{V}), \quad \chi \mapsto \partial_{u_{i}^{\alpha}} \chi$

2) $\iota_{\alpha}^{i}=\partial_{\left.u_{i}^{\alpha}\right\lrcorner:} \Omega^{p}(\mathbf{V}) \rightarrow \Omega^{p-1}(\mathbf{V}), \quad \chi \mapsto \iota_{\alpha}^{i} \chi$,

3) $\varepsilon_{i}^{\alpha}=d_{V} u_{i}^{\alpha} \wedge: \Omega^{p}(\mathbf{V}) \rightarrow \Omega^{p+1}(\mathbf{V}), \quad \chi \mapsto \varepsilon_{i}^{\alpha} \chi$,

где

$$
\begin{aligned}
\chi & =\frac{1}{p !} \sum_{|\boldsymbol{\alpha}, \mathbf{i}|=p} \chi_{\boldsymbol{\alpha}}^{\mathbf{i}} \cdot d_{V} u_{\mathbf{i}}^{\boldsymbol{\alpha}} \in \Omega^{p}(\mathbf{V}), & \partial_{u_{i}^{\alpha}} \chi & =\frac{1}{p !} \sum_{|\boldsymbol{\alpha}, \mathbf{i}|=p}\left(\partial_{u_{i}^{\alpha}} \chi_{\boldsymbol{\alpha}}^{\mathbf{i}}\right) \cdot d_{V} u_{\mathbf{i}}^{\boldsymbol{\alpha}}, \\
\iota_{\alpha}^{i} \chi & =\frac{1}{(p-1) !} \sum_{|\boldsymbol{\alpha}, \mathbf{i}|=p-1} \chi_{\alpha \boldsymbol{\alpha}}^{i \mathbf{i}} \cdot d_{V} u_{\mathbf{i}}^{\boldsymbol{\alpha}}, & \varepsilon_{i}^{\alpha} \chi & =\frac{1}{p !} \sum_{|\boldsymbol{\alpha}, \mathbf{i}|=p} \chi_{\boldsymbol{\alpha}}^{\mathbf{i}} \cdot d_{V} u_{i \mathbf{i}}^{\alpha \boldsymbol{\alpha}},
\end{aligned}
$$

во второй строке слева $\alpha \boldsymbol{\alpha}=\left(\alpha, \alpha_{1}, \ldots, \alpha_{p-1}\right), i \mathbf{i}=\left(i, i_{1}, \ldots, i_{p-1}\right)$, а справа $\alpha \boldsymbol{\alpha}=$ $\left(\alpha, \alpha_{1}, \ldots, \alpha_{p}\right), i \mathbf{i}=\left(i, i_{1}, \ldots, i_{p}\right)$.

Вертикалъный дифференциал действует по формуле

$$
d_{V}=d_{V}^{p}=\sum_{\alpha, i} \varepsilon_{i}^{\alpha} \circ \partial_{u_{i}^{\alpha}}: \Omega^{p}(\mathbf{V}) \rightarrow \Omega^{p+1}(\mathbf{V}),
$$


так что

$$
\begin{aligned}
\chi=\frac{1}{p !} \sum_{|\boldsymbol{\alpha}, \mathbf{i}|=p} \chi_{\boldsymbol{\alpha}}^{\mathbf{i}} \cdot d_{V} u_{\mathbf{i}}^{\boldsymbol{\alpha}} \quad \mapsto \quad d_{V} \chi=\frac{1}{(p+1) !} \sum_{|\boldsymbol{\alpha}, \mathbf{i}|=p+1}\left(d_{V} \chi\right)_{\boldsymbol{\alpha}}^{\mathbf{i}} \cdot d_{V} u_{\mathbf{i}}^{\boldsymbol{\alpha}}, \\
\left(d_{V} \chi\right)_{\boldsymbol{\alpha}}^{\mathbf{i}}=\sum_{0 \leqslant r \leqslant p}(-1)^{r} \partial_{u_{i_{r}}^{\alpha_{r}}} \chi_{\boldsymbol{\alpha}^{r}}^{\mathbf{i}^{r}},
\end{aligned}
$$

здесь $\boldsymbol{\alpha}^{r}=\left(\alpha_{0}, \ldots, \widetilde{\alpha_{r}}, \ldots, \alpha_{p}\right), \mathbf{i}^{r}=\left(i_{0}, \ldots, \widetilde{i_{r}}, \ldots, i_{p}\right)$, индекс под знаком " $\sim "$ пропускается. В частности, $d_{V}-$ косое дифференцирование внешней алгебры $\Omega(\mathbf{V})$.

Заметим, что вертикальный дифференциал функции $f(\mathbf{u})=u_{i}^{\alpha} \in \mathcal{A}(\mathbf{V})=\Omega^{0}(\mathbf{V})$ есть $d_{V} u_{i}^{\alpha}$ в соответствии с введенными выше обозначениями.

ПРЕДЛОЖЕНИЕ 6. Справедливы стандартные равенства:

$$
\begin{aligned}
& {\left[d_{V}, \partial_{u_{i}^{\alpha}}\right]=0,} \\
& d_{V} \circ \iota_{\alpha}^{i}+\iota_{\alpha}^{i} \circ d_{V}=\partial_{u_{i}^{\alpha}}, \\
& d_{V} \circ \varepsilon_{i}^{\alpha}+\varepsilon_{i}^{\alpha} \circ d_{V}=0, \\
& d_{V} \circ d_{V}=0
\end{aligned}
$$

для всех $\alpha \in \mathrm{A}, i \in \mathbb{L}$.

Доказательство проводится прямыми вычислениями.

В силу (4) определен вертикальный комплекс $\left\{\Omega(\mathbf{V}) ; d_{V}\right\}$ - комплекс де Рама бесконечномерного пространства $\mathbf{V} \simeq \mathbb{R}_{\mathbb{L}}^{\mathrm{A}}$.

Теорема 1. Пополненный комплекс де Рама пространства $\mathbf{V}$

$$
0 \longrightarrow \mathbb{R} \longrightarrow \Omega^{0}(\mathbf{V}) \stackrel{d_{V}}{\longrightarrow} \Omega^{1}(\mathbf{V}) \stackrel{d_{V}}{\longrightarrow} \Omega^{2}(\mathbf{V}) \stackrel{d_{V}}{\longrightarrow} \Omega^{3}(\mathbf{V}) \stackrel{d_{V}}{\longrightarrow} \ldots
$$

точный, т.е. все его пространства когомологий тривиальные.

ДокАЗАтЕльство. Действительно, в данном случае справедлива гомотопическая формула Пуанкаре (см., например, [5]; то, что пространство V бесконечномерно, несущественно, поскольку каждая компонента $\chi_{\boldsymbol{\alpha}}^{\mathbf{i}}$ зависит лишь от конечного числа переменных $u_{i}^{\alpha}$ и число ненулевых компонент тоже конечно):

$$
d_{V} \circ h_{V}+h_{V} \circ d_{V}=\mathrm{id}-\varrho_{V},
$$

где гомотопический оператор $h_{V}=h_{V}^{p}: \Omega^{p}(\mathbf{V}) \rightarrow \Omega^{p-1}(\mathbf{V})$ действует по правилу

$$
\begin{gathered}
h_{V}^{0}=0, \\
\chi=\frac{1}{p !} \sum_{|\boldsymbol{\alpha}, \mathbf{i}|=p} \chi_{\boldsymbol{\alpha}}^{\mathbf{i}} \cdot d_{V} u_{\mathbf{i}}^{\boldsymbol{\alpha}} \mapsto h_{V}^{p} \chi=\frac{1}{(p-1) !} \sum_{|\boldsymbol{\alpha}, \mathbf{i}|=p-1}\left(h_{V}^{p} \chi\right)_{\boldsymbol{\alpha}}^{\mathbf{i}} \cdot d_{V} u_{\mathbf{i}}^{\boldsymbol{\alpha}}, \\
\left(h_{V}^{p} \chi\right)_{\boldsymbol{\alpha}}^{\mathbf{i}}(\mathbf{u})=\int_{0}^{1} t^{p-1} \sum_{\alpha, i} u_{i}^{\alpha} \chi_{\alpha \boldsymbol{\alpha}}^{i \mathbf{i}}(t \mathbf{u}) d t, \quad p \in \mathbb{N},
\end{gathered}
$$

а линейное отображение $\varrho_{V}=\varrho_{V}^{p}: \Omega^{p}(\mathbf{V}) \rightarrow \Omega^{p}(\mathbf{V})$ действует по правилу

$$
\begin{array}{lll}
f \mapsto \varrho_{V}^{0} f, & \left(\varrho_{V}^{0} f\right)(\mathbf{u})=f(\mathbf{0}), & \\
\varrho_{V}^{p}=0, & & p \in \mathbb{N} .
\end{array}
$$


2.2. Горизонтальный разностный комплекс. Определена градуированная внешняя алгебра разностных форм

$$
\Omega(\mathbb{L})=\Omega(\mathcal{A}(\mathbb{L}))=\bigoplus_{0 \leqslant q \leqslant D} \Omega^{q}(\mathbb{L}),
$$

где $\mathcal{A}(\mathbb{L})$-модули

$$
\Omega^{q}(\mathbb{L})=\Omega^{q}(\mathcal{A}(\mathbb{L}))=\operatorname{Hom}_{\mathcal{A}(\mathbb{L})}\left(\wedge_{\mathcal{A}(\mathbb{L})}^{q} \mathfrak{D}(\mathbb{L}) ; \mathcal{A}(\mathbb{L})\right)=\wedge_{\mathcal{A}(\mathbb{L})}^{q} \mathfrak{D}^{*}(\mathbb{L}) .
$$

Положим $\boldsymbol{\mu}=\left(\mu_{1}, \ldots, \mu_{q}\right) \in \mathbb{D}^{q}$ и будем в этом случае писать $|\boldsymbol{\mu}|=q$. В таких обозначениях

$$
\Omega^{q}(\mathbb{L})=\left\{\varrho=\frac{1}{q !} \sum_{|\boldsymbol{\mu}|=q} \varrho_{\boldsymbol{\mu}} \cdot d_{H} i^{\mu} \mid \varrho_{\boldsymbol{\mu}} \in \mathcal{A}(\mathbb{L})\right\}
$$

где:

1) $\varrho_{\sigma(\boldsymbol{\mu})}=\operatorname{sign}(\sigma) \varrho_{\boldsymbol{\mu}}$ для всех перестановок $\sigma \in \Sigma_{q}$,

2) $d_{H} i^{\mu}=d_{H} i^{\mu_{1}} \wedge \cdots \wedge d_{H} i^{\mu_{q}}$.

По аналогии с вертикальным комплексом для всех $i \in \mathbb{L}, \mu \in \mathbb{D}$ определены следующие линейные отображения:

1) $T_{i}: \Omega^{q}(\mathbb{L}) \rightarrow \Omega^{q}(\mathbb{L}), \quad \varrho \mapsto T_{i} \varrho$,

2) $\Delta_{\mu}: \Omega^{q}(\mathbb{L}) \rightarrow \Omega^{q}(\mathbb{L}), \quad \varrho \mapsto \Delta_{\mu} \varrho$,

3) $\left.\iota_{\mu}: \Omega^{q}(\mathbb{L}) \rightarrow \Omega^{q-1}(\mathbb{L}), \quad \varrho \mapsto \iota_{\mu} \varrho=\Delta_{\mu}\right\lrcorner \varrho$,

4) $\varepsilon^{\mu}: \Omega^{q}(\mathbb{L}) \rightarrow \Omega^{q+1}(\mathbb{L}), \quad \varrho \mapsto d_{H} i^{\mu} \wedge \varrho$,

где

$$
\begin{aligned}
\varrho & =\frac{1}{q !} \sum_{|\boldsymbol{\mu}|=q} \varrho_{\boldsymbol{\mu}} \cdot d_{H} i^{\boldsymbol{\mu}}, & & \\
T_{i} \varrho & =\frac{1}{q !} \sum_{|\boldsymbol{\mu}|=q}\left(T_{i} \varrho_{\boldsymbol{\mu}}\right) \cdot d_{H} i^{\boldsymbol{\mu}}, & \Delta_{\mu} \varrho & =\frac{1}{q !} \sum_{|\boldsymbol{\mu}|=q}\left(\Delta_{\mu} \varrho_{\boldsymbol{\mu}}\right) \cdot d_{H} i^{\boldsymbol{\mu}}, \\
\iota_{\mu} \varrho & =\frac{1}{(q-1) !} \sum_{|\boldsymbol{\mu}|=q-1} \varrho_{\mu \boldsymbol{\mu}} \cdot d_{H} i^{\boldsymbol{\mu}}, & \varepsilon^{\mu} \varrho & =\frac{1}{q !} \sum_{|\boldsymbol{\mu}|=q} \varrho_{\boldsymbol{\mu}} \cdot d_{H} i^{\mu \boldsymbol{\mu}},
\end{aligned}
$$

здесь слева $\mu \boldsymbol{\mu}=\left(\mu, \mu_{1}, \ldots, \mu_{q-1}\right)$, а справа $\mu \boldsymbol{\mu}=\left(\mu, \mu_{1}, \ldots, \mu_{q}\right)$.

Заметим, что отображения $\iota_{\mu}$ суть косые дифференцирования внешней алгебры $\Omega(\mathbb{L})$.

Горизонтальный дифференииал действует по формуле

$$
d_{H}=d_{H}^{q}=\sum_{\mu} \varepsilon^{\mu} \circ \Delta_{\mu}: \Omega^{q}(\mathbb{L}) \rightarrow \Omega^{q+1}(\mathbb{L}),
$$

так что

$$
\begin{aligned}
\varrho=\frac{1}{q !} \sum_{|\boldsymbol{\mu}|=q} \varrho_{\boldsymbol{\mu}} \cdot d_{H} i^{\boldsymbol{\mu}} \mapsto \quad d_{H} \varrho & =\frac{1}{(q+1) !} \sum_{|\boldsymbol{\mu}|=q+1}\left(d_{H} \varrho\right)_{\boldsymbol{\mu}} \cdot d_{H} i^{\boldsymbol{\mu}}, \\
\left(d_{H} \varrho\right)_{\boldsymbol{\mu}} & =\sum_{0 \leqslant r \leqslant q}(-1)^{r} \Delta_{\mu_{r}} \varrho_{\boldsymbol{\mu}^{r}},
\end{aligned}
$$


здесь $\boldsymbol{\mu}^{r}=\left(\mu_{0}, \ldots, \widetilde{\mu_{r}}, \ldots, \mu_{q}\right)$, индекс под знаком “ $\sim$ пропускается.

Заметим, что горизонтальный дифференциал функции $f(i)=i^{\mu} \in \mathcal{A}(\mathbb{L})=\Omega^{0}(\mathbb{L})$ есть $d_{H} i^{\mu}$ в соответствии с введенными выше обозначениями.

ПреДЛОЖЕНИЕ 7. Справедливы следующие равенства:

$$
\begin{aligned}
& {\left[d_{H}, T_{i}\right]=\left[d_{H}, \Delta_{\mu}\right]=0,} \\
& d_{H} \circ \iota_{\mu}+\iota_{\mu} \circ d_{H}=\Delta_{\mu}, \\
& d_{H} \circ \varepsilon^{\mu}+\varepsilon^{\mu} \circ d_{H}=0, \\
& d_{H} \circ d_{H}=0
\end{aligned}
$$

для всех $i \in \mathbb{L}, \mu \in \mathbb{D}$.

Доказательство проводится прямыми вычислениями.

Равенство (6) имеет следующее обобщение.

ПреДЛОЖЕНИЕ 8. Для каждой пары $i \in \mathbb{L}, q \in \mathbb{Z}_{+}$существует линейное отображение $\mathrm{I}_{i}=\mathrm{I}_{i}^{q}: \Omega^{q}(\mathbb{L}) \rightarrow \Omega^{q-1}(\mathbb{L})$ такое, что

$$
d_{H} \circ \mathrm{I}_{i}+\mathrm{I}_{i} \circ d_{H}=T_{i}-\mathrm{id} .
$$

ДокАЗАТЕЛЬСтво. Соединим точку $0 \in \mathbb{L}$ с точкой $i=\sum_{\mu \in \mathbb{D}} i^{\mu} e_{\mu}$ стандартной целочисленной ломаной с вершинами

$$
i_{0}=0, i_{\nu}=i_{\nu-1}+i^{\nu} e_{\nu}, \nu \in \mathbb{D}
$$

и зададим линейные отображения $S_{i}^{\nu}=\left(S_{i}^{\nu}\right)^{q}: \Omega^{q}(\mathbb{L}) \rightarrow \Omega^{q}(\mathbb{L}), \nu \in \mathbb{D}$, правилом

$$
S_{i}^{\nu}= \begin{cases}\sum_{0 \leqslant r<i^{\nu}} T_{i_{\nu-1}+r e_{\nu}}, & i^{\nu}>0, \\ 0, & i^{\nu}=0, \\ -\sum_{i^{\nu} \leqslant r<0} T_{i_{\nu-1}+r e_{\nu}}, & i^{\nu}<0 .\end{cases}
$$

Очевидно, $\left[\Delta_{\mu}, S_{i}^{\nu}\right]=0$ для всех $\mu, \nu \in \mathbb{D}$, и легко проверяется, что $S_{i}^{\nu} \circ \Delta_{\nu}=T_{i_{\nu}}-$ $T_{i_{\nu-1}}$ для всех $\nu \in \mathbb{D}$. Более того, в силу равенств (5) и (6) линейное отображение $\mathrm{I}_{i}=\sum_{\nu \in \mathbb{D}} S_{i}^{\nu} \circ \iota_{\nu}$ удовлетворяет требуемому соотношению (9).

В силу (8) определен горизонтальный комплекс $\left\{\Omega(\mathbb{L}) ; d_{H}\right\}$ - разностный комплекс решетки $\mathbb{L}$.

ТеОрема 2. Пополненный разностный комплекс решетки $\mathbb{L}$

$$
0 \longrightarrow \mathbb{R} \longrightarrow \Omega^{0}(\mathbb{L}) \stackrel{d_{H}}{\longrightarrow} \Omega^{1}(\mathbb{L}) \stackrel{d_{H}}{\longrightarrow} \ldots \stackrel{d_{H}}{\longrightarrow} \Omega^{D}(\mathbb{L}) \longrightarrow 0
$$

точный, т.е. все его пространства когомологий тривиальные. 
ДокАзАтЕльство. Эта теорема доказана в работе [6], однако ради полноты изложения приведем доказательство здесь. Зададим линейные отображения $S^{\nu}: \mathcal{A}(\mathbb{L}) \rightarrow$ $\mathcal{A}(\mathbb{L}), f \mapsto S^{\nu} f$, правилом $\left(S^{\nu} f\right)(i)=\left(S_{i}^{\nu} f\right)(0)$ (см. доказательство предложения 8), $\nu \in \mathbb{D}$. Легко проверяется, что

$$
\left(\Delta_{\mu} S^{\nu} f\right)(i)= \begin{cases}\left(S^{\nu} \Delta_{\mu} f\right)(i), & \mu<\nu \\ f\left(i_{\mu}\right), & \mu=\nu \\ 0, & \mu>\nu\end{cases}
$$

для всех $\mu, \nu \in \mathbb{D}$ и

$$
\left(S^{\mu} \Delta_{\mu} f\right)(i)=\left.f\right|_{i_{\mu-1}} ^{i_{\mu}}=f\left(i_{\mu}\right)-f\left(i_{\mu-1}\right)
$$

для всех $\mu \in \mathbb{D}$.

Определим гомотопический оператор $h_{H}=h_{H}^{q}: \Omega^{q}(\mathbb{L}) \rightarrow \Omega^{q-1}(\mathbb{L})$ правилом

$$
\begin{aligned}
h_{H}^{0} & =0, \\
\varrho & =\frac{1}{q !} \sum_{|\boldsymbol{\mu}|=q} \varrho_{\boldsymbol{\mu}} \otimes d_{H} i^{\boldsymbol{\mu}} \mapsto h_{H}^{q} \varrho=\frac{1}{(q-1) !} \sum_{|\boldsymbol{\mu}|=q-1}\left(h_{H}^{q} \varrho\right)_{\boldsymbol{\mu}} \otimes d_{H} i^{\boldsymbol{\mu}},
\end{aligned}
$$

где

$$
\left(h_{H}^{q} \varrho\right)_{\boldsymbol{\mu}}(i)=\sum_{\nu>\boldsymbol{\mu}}\left(S^{\nu} \varrho_{\nu \boldsymbol{\mu}}\right)(i), \quad i \in \mathbb{L}, \quad 1 \leqslant q \leqslant D,
$$

$\boldsymbol{\mu}=\left(\mu_{1}, \ldots, \mu_{q-1}\right), \nu \boldsymbol{\mu}=\left(\nu, \mu_{1}, \ldots, \nu_{q-1}\right)$, запись $\nu>\boldsymbol{\mu}$ означает, что $\nu>\mu_{r}$ для всех $1 \leqslant r \leqslant q-1$.

Линейное отображение $R_{H}=R_{H}^{q}: \Omega^{q}(\mathbb{L}) \rightarrow \Omega^{q}(\mathbb{L})$ определим правилом

$$
\begin{aligned}
& \left(R_{H}^{0} f\right)(i)=f(0) \quad \text { для всех } f \in \mathcal{A}(\mathbb{L})=\Omega^{0}(\mathbb{L}), \quad i, 0 \in \mathbb{L}, \\
& R_{H}^{q}=0, \quad 1 \leqslant q \leqslant D .
\end{aligned}
$$

Справедлива гомотопическая формула

$$
d_{H} \circ h_{H}+h_{H} \circ d_{H}=\mathrm{id}-R_{H} .
$$

Действительно, пусть

$$
\varrho=\frac{1}{q !} \sum_{|\boldsymbol{\mu}|=q} \varrho_{\boldsymbol{\mu}} \otimes d_{H} i^{\mu} \in \Omega^{q}(\mathbb{L}), \quad \varrho_{\boldsymbol{\mu}} \in \mathcal{A}(\mathbb{L}), \quad 1 \leqslant q \leqslant D .
$$

Тогда, с одной стороны,

$$
\begin{gathered}
\left(d_{H} h_{H} \varrho\right)_{\boldsymbol{\mu}}(i)=\sum_{1 \leqslant r \leqslant q}(-1)^{r-1} \Delta_{\mu_{r}}\left(h_{H} \varrho\right)_{\boldsymbol{\mu}^{r}}(i)=\sum_{1 \leqslant r \leqslant q}(-1)^{r-1} \Delta_{\mu_{r}} \sum_{\nu>\boldsymbol{\mu}^{r}}\left(S^{\nu} \varrho_{\nu \boldsymbol{\mu}^{r}}\right)(i)= \\
=(-1)^{r_{\max }-1} \Delta_{\mu_{\max }} \sum_{\nu \geqslant \mu_{\max }}\left(S^{\nu} \varrho_{\nu \boldsymbol{\mu}^{r_{\max }}}\right)(i)+\sum_{r \neq r_{\max }}(-1)^{r-1} \Delta_{\mu_{r}} \sum_{\nu>\mu_{\max }}\left(S^{\nu} \varrho_{\nu \boldsymbol{\mu}^{r}}\right)(i)= \\
=\varrho_{\boldsymbol{\mu}}\left(i_{\mu_{\max }}\right)+\sum_{1 \leqslant r \leqslant q}(-1)^{r-1} \sum_{\nu>\mu_{\max }}\left(S^{\nu} \Delta_{\mu_{r}} \varrho_{\nu \boldsymbol{\mu}^{r}}\right)(i),
\end{gathered}
$$


где $\mu_{\max }=\mu_{r_{\max }}>\mu_{r}$ для всех $1 \leqslant r \leqslant q, r \neq r_{\max }$. С другой стороны,

$$
\begin{aligned}
\left(h_{H} d_{H} \varrho\right)_{\boldsymbol{\mu}}(i) & =\sum_{\nu>\boldsymbol{\mu}}\left(S^{\nu}\left(d_{H} \varrho\right)_{\nu \boldsymbol{\mu}}\right)(i)= \\
& =\sum_{\nu>\mu_{\max }} S^{\nu}\left(\Delta_{\nu} \varrho_{\boldsymbol{\mu}}+\sum_{1 \leqslant r \leqslant q}(-1)^{r} \Delta_{\mu_{r}} \varrho_{\nu \boldsymbol{\mu}^{r}}\right)(i)= \\
& =\left.\varrho_{\boldsymbol{\mu}}\right|_{i_{\mu_{\max }}} ^{i}+\sum_{\nu>\mu_{\max }} \sum_{1 \leqslant r \leqslant q}(-1)^{r}\left(S^{\nu} \Delta_{\mu_{r}} \varrho_{\nu \boldsymbol{\mu}^{r}}\right)(i) .
\end{aligned}
$$

Складывая полученные представления, убеждаемся в справедливости гомотопической формулы для $1 \leqslant q \leqslant D$. Случай $q=0$ проверяется элементарно.

2.3. Дифференциально-разностный бикомплекс. Определена биградуированная внешняя алгебра дифференциально-разностных форм

$$
\Omega(\mathbf{J})=\Omega(\mathcal{A}(\mathbf{J}))=\bigoplus_{p \in \mathbb{Z}_{+}, 0 \leqslant q \leqslant D} \Omega^{p q}(\mathbf{J}), \quad \Omega^{p q}(\mathbf{J})=\Omega^{p}(\mathbf{V}) \wedge_{\mathbb{R}} \Omega^{q}(\mathbb{L}),
$$

где $\mathcal{A}(\mathbf{J})$-модули $\Omega^{p q}(\mathbf{J})$ имеют представления

$$
\begin{aligned}
\Omega^{p q}(\mathbf{J}) & =\left\{\omega=\frac{1}{q !} \sum_{|\boldsymbol{\mu}|=q} \chi_{\boldsymbol{\mu}} \wedge d_{H} i^{\boldsymbol{\mu}} \mid \chi_{\boldsymbol{\mu}} \in \mathcal{A}(\mathbb{L}) \otimes_{\mathbb{R}} \Omega^{p}(\mathbf{V})\right\}= \\
& =\left\{\omega=\frac{1}{p !} \sum_{|\boldsymbol{\alpha}, \mathbf{i}|=p} d_{V} u_{\mathbf{i}}^{\boldsymbol{\alpha}} \wedge \varrho_{\boldsymbol{\alpha}}^{\mathbf{i}} \mid \varrho_{\boldsymbol{\alpha}}^{\mathbf{i}} \in \mathcal{A}(\mathbf{V}) \otimes_{\mathbb{R}} \Omega^{q}(\mathbb{L})\right\}= \\
& =\left\{\omega=\frac{1}{p ! q !} \sum_{|\boldsymbol{\alpha}, \mathbf{i}|=p} \sum_{|\boldsymbol{\mu}|=q} \omega_{\mathbf{i}, \boldsymbol{\mu}}^{\boldsymbol{\alpha}} \cdot d_{V} u_{\mathbf{i}}^{\boldsymbol{\alpha}} \wedge d_{H} i^{\boldsymbol{\mu}} \mid \omega_{\mathbf{i}, \boldsymbol{\mu}}^{\boldsymbol{\alpha}} \in \mathcal{A}(\mathbf{J})\right\} .
\end{aligned}
$$

Все вертикальные операции, определенные выше на алгебре вертикальных форм $\Omega(\mathbf{V})$, переносятся на алгебру $\Omega(\mathbf{J})$ тривиальным образом:

$$
L(\chi \wedge \varrho)=(L \chi) \wedge \varrho, \quad L=\partial_{u_{i}^{\alpha}}, \iota_{\alpha}^{i}, \varepsilon_{i}^{\alpha}, d_{V},
$$

для всех $\chi \in \Omega(\mathbf{V}), \varrho \in \Omega(\mathbb{L})$. В свою очередь, сдвиги $T_{i}, i \in \mathbb{L}$, определяются как морфизмы алгебры $\Omega(\mathbf{J})$ :

$$
T_{i}\left(\omega_{1} \wedge \omega_{2}\right)=\left(T_{i} \omega_{1}\right) \wedge\left(T_{i} \omega_{2}\right) \quad \text { для всех } \quad \omega_{1}, \omega_{2} \in \Omega(\mathbf{J}),
$$

причем

$$
T_{i}(f)=f \circ T_{i}, \quad T_{i}\left(d_{V} u_{j}^{\alpha}\right)=d_{V} u_{j+i}^{\alpha}, \quad T_{i}\left(d_{H} i^{\mu}\right)=d_{H} i^{\mu}
$$

для всех $i, j \in \mathbb{L}, f \in \mathcal{A}(\mathbf{J}), \alpha \in \mathrm{A}, \mu \in \mathbb{D}$. Эти правила полностью определяют действие на алгебре $\Omega(\mathbf{J})$ горизонтальных операций $\Delta_{\mu}, \iota_{\mu}$, $\varepsilon^{\mu}$ и $d_{H}$. Например, $d_{H} u_{i}^{\alpha}=\sum_{\mu} \Delta_{\mu} u_{i}^{\alpha} \cdot d_{H} i^{\mu}, \Delta_{\mu} u_{i}^{\alpha}=u_{i+e_{\mu}}^{\alpha}-u_{i}^{\alpha}$.

По-прежнему справедливы равенства $d_{V} \circ d_{V}=0, d_{H} \circ d_{H}=0$, и, кроме того, выполняется связующее равенство $d_{V} \circ d_{H}+d_{H} \circ d_{V}=0$, так что определен бикомплекс $\left\{\Omega(\mathbf{J}) ; d_{V}, d_{H}\right\}-$-ифференииально-разностный бикомплекс пространства разностных джетов $\mathbf{J}$. 


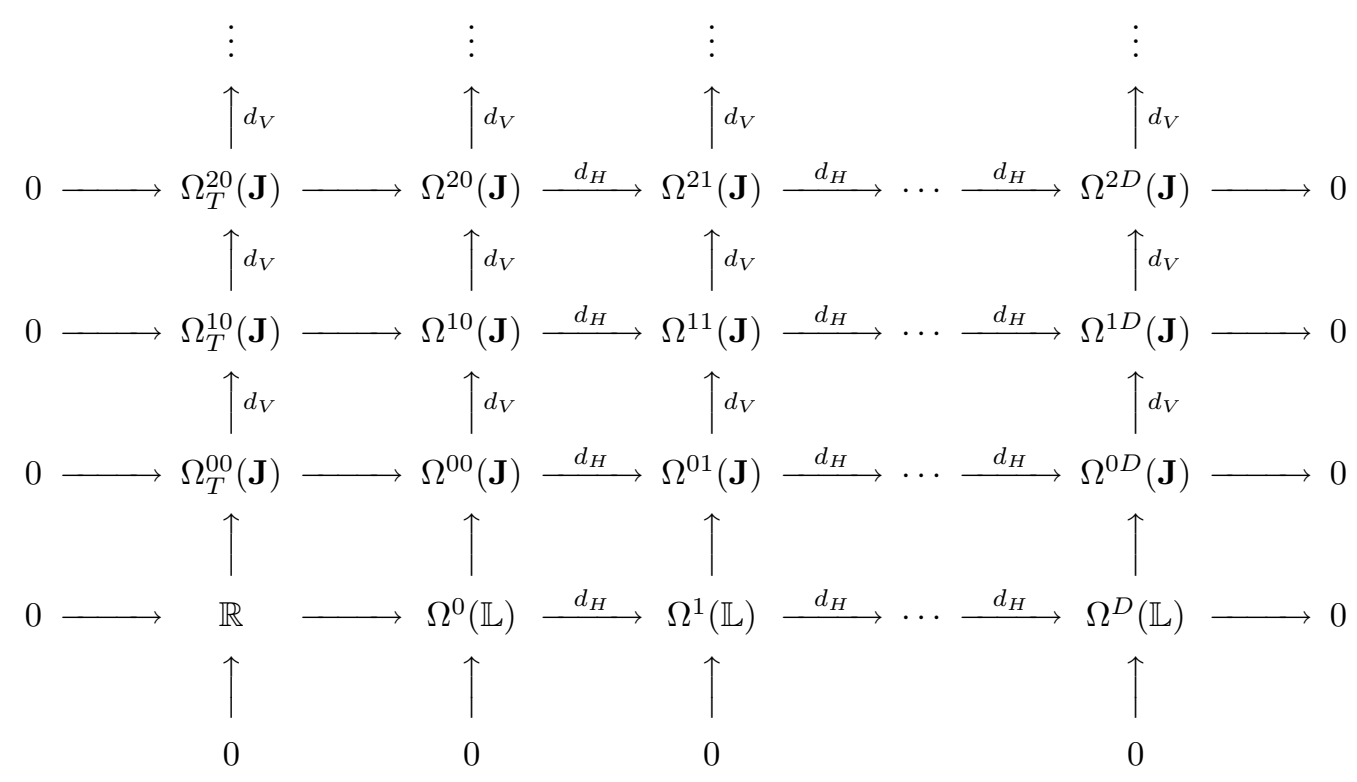

Рис. 1. Пополненный бикомплекс на пространстве $\mathbf{J}$.

Нам еще понадобится подалгебра инвариантных форм (внешняя алгебра дифференциально-разностных форм, инвариантных относительно сдвигов)

$$
\Omega_{T}(\mathbf{J})=\left\{\omega \in \Omega(\mathbf{J}) \mid T_{i} \omega=\omega \text { для всех } i \in \mathbb{L}\right\} .
$$

Алгебра $\Omega_{T}^{00}(\mathbf{J})$, т. е. инвариантные функции на пространстве $\mathbf{J}$, описана выше (см. предложение 3), полное описание инвариантных форм будет дано ниже (см. предложение 10).

Теорема 3. Справедливы следующие утверждения:

1) вертикальные дифберенииаль имеют сужения

$$
d_{V}=d_{V}^{p q}: \Omega_{T}^{p q}(\mathbf{J}) \rightarrow \Omega_{T}^{p+1, q}(\mathbf{J}), \quad p \in \mathbb{Z}_{+}, \quad 0 \leqslant q \leqslant D
$$

2) определен пополненный бикомплекс пространства разностных джетов, изображенныци на рис. 1;

3) пополненный бикомплекс пространства разностных джетов ациклический, m.е. все его строки и столбцы точные.

Отдельные утверждения этой теоремы можно доказать непосредственно в данной постановке, однако полное доказательство естественно проводить в специальной системе координат. Эта система координат введена в п. 2.4, там же дано и доказательство данной теоремы (см. следствие 2). Заметим, что в случае стандартного вариационного бикомплекса евклидова пространства $\mathbb{R}^{D}$ такая система координат отсутствует, чем и объясняется различие свойств бикомплекса для решетки и для евклидова пространства. 
2.4. Факторизующее представление. Пусть $\widetilde{\mathbf{V}}$ - копия пространства V с координатами $\tilde{\mathbf{u}}=\left(\tilde{u}_{l}^{\alpha}\right)$, а $\tilde{\mathbf{J}}-$ копия пространства $\mathbf{J}$ с координатами $(k, \tilde{\mathbf{u}})$. Введем замену переменных

$$
\varkappa: \tilde{\mathbf{J}} \simeq \mathbf{J}, \quad(k, \tilde{\mathbf{u}}) \mapsto \varkappa(k, \tilde{\mathbf{u}})=(k, \mathbf{u}), \quad \mathbf{u}=T_{k} \tilde{\mathbf{u}}, \quad \tilde{\mathbf{u}}=T_{-k} \mathbf{u},
$$

или подробнее $u_{l}^{\alpha}=\tilde{u}_{l+k}^{\alpha}, \tilde{u}^{\alpha}=u_{l-k}^{\alpha}$ для всех $\alpha \in \mathrm{A}, k, l \in \mathbb{L}$.

При такой замене

$$
T_{i}: \mathbf{J} \simeq \mathbf{J} \mapsto \widetilde{T}_{i}: \tilde{\mathbf{J}} \simeq \tilde{\mathbf{J}}, \quad \widetilde{T}_{i}=\varkappa^{-1} \circ T_{i} \circ \varkappa,
$$

или подробнее $(k, \tilde{\mathbf{u}}) \mapsto \widetilde{T}_{i}(k, \tilde{\mathbf{u}})=\left(T_{i} k, \tilde{\mathbf{u}}\right)$ для всех $(k, \tilde{\mathbf{u}}) \in \tilde{\mathbf{J}}, i \in \mathbb{L}$. Таким образом, в новой системе координат полнье сдвиги действуют лишь на иелочисленнье переменные $k \in \mathbb{L}$.

Для расслоений соответственно имеем

$$
\boldsymbol{\pi}: \mathbf{J} \rightarrow \mathbb{L} \mapsto \tilde{\boldsymbol{\pi}}: \tilde{\mathbf{J}} \rightarrow \mathbb{L}, \quad \tilde{\boldsymbol{\pi}}=\boldsymbol{\pi} \circ \varkappa, \quad(k, \tilde{\mathbf{u}}) \mapsto k,
$$

а для сечений имеем изоморфизм

$$
\varkappa \circ: \mathcal{M}(\mathbb{L} ; \tilde{\mathbf{J}}) \simeq \mathcal{M}(\mathbb{L} ; \mathbf{J}), \quad \widetilde{\phi} \mapsto \phi=\varkappa \circ \widetilde{\phi}
$$

так что $\phi(k)=T_{k} \widetilde{\boldsymbol{\phi}}(k)$, подробнее $\varphi_{l}^{\alpha}(k)=\widetilde{\varphi}_{l+k}^{\alpha}(k)$ для всех $\widetilde{\boldsymbol{\phi}} \in \mathcal{M}(\mathbb{L} ; \tilde{\mathbf{J}}), k, l \in \mathbb{L}$, $\alpha \in \mathrm{A}$.

Действие $T: \mathbb{L} \rightarrow \operatorname{Aut}(\mathcal{M}(\mathbb{L} ; \mathbf{V}))$ индуцирует соответствующее действие $\widetilde{T}: \mathbb{L} \rightarrow$ $\operatorname{Aut}(\mathcal{M}(\mathbb{L} ; \tilde{\mathbf{V}}))$ :

$$
\widetilde{\phi} \mapsto \widetilde{T}_{i} \widetilde{\boldsymbol{\phi}}=\varkappa^{-1} \circ T_{i} \circ \varkappa \circ \widetilde{\boldsymbol{\phi}}, \quad\left(\widetilde{T}_{i} \widetilde{\boldsymbol{\phi}}\right)_{l}^{\alpha}(k)=\widetilde{\varphi}_{l}^{\alpha}(k+i),
$$

для всех $\widetilde{\phi}=\left(\widetilde{\varphi}_{l}^{\alpha}(k)\right), i, k, l \in \mathbb{L}, \alpha \in \mathrm{A}$. Образно говоря, здесъ сдвиги действуют послойно.

Отображение джетов $\mathbf{j}: \mathcal{M}(\mathbb{L} ; V) \rightarrow \mathcal{M}(\mathbb{L} ; \mathbf{V})$ индуцирует соответствующее отображение

$$
\tilde{\mathbf{j}}: \mathcal{M}(\mathbb{L} ; V) \rightarrow \mathcal{M}(\mathbb{L} ; \tilde{\mathbf{V}}), \quad \tilde{\mathbf{j}}=\varkappa^{-1} \circ \mathbf{j},
$$

т. е. $(\tilde{\mathbf{j}} \varphi)(k)=\varkappa^{-1}(\mathbf{j} \varphi(k))$, подробнее $(\tilde{\mathbf{j}} \varphi)_{l}(k)=(\mathbf{j} \varphi)_{l-k}(k)=\varphi(l)$, или коротко

$$
(\tilde{\mathbf{j}} \varphi)(k)=(\mathbf{j} \varphi)(0) \quad \text { для всех } \quad \varphi \in \mathcal{M}(\mathbb{L} ; V), k \in \mathbb{L} .
$$

Таким образом, в новых координатах предложение 1 принимает следующий вид.

ПрЕДЛОЖЕНИЕ 9. Имеют место следующие утверждения.

1. $\widetilde{T}_{i} \circ \tilde{\mathbf{j}}=\tilde{\mathbf{j}}$ для всех $i \in \mathbb{L}$.

2. Сечение $\widetilde{\boldsymbol{\phi}}=\left(\widetilde{\varphi}_{l}^{\alpha}(k)\right) \in \mathcal{M}(\mathbb{L} ; \tilde{\mathbf{V}})$ есть джет некоторого сечения $\varphi=\left(\varphi^{\alpha}(k)\right) \in$ $\mathcal{M}(\mathbb{L} ; V)$, m.е. $\widetilde{\boldsymbol{\phi}}=\tilde{\mathbf{j}} \varphi$, тогда и только тогда, когда $T_{i} \widetilde{\boldsymbol{\phi}}=\widetilde{\boldsymbol{\phi}}$ для всех $i \in \mathbb{L}$, m.е. когда $\widetilde{\varphi}_{l}^{\alpha}(k)=\widetilde{\varphi}_{l}^{\alpha}($ не зависит от $k)$, и в этом случае $\varphi^{\alpha}(k)=\widetilde{\varphi}_{k}^{\alpha}$.

3. Для каждой точки $\tilde{\mathbf{u}} \in \tilde{\mathbf{V}}$ существует одно и только одно сечение $\varphi=$ $\left(\varphi^{\alpha}(k)\right) \in \mathcal{M}(\mathbb{L} ; V)$ такое, ито $\tilde{\mathbf{j}} \varphi=\tilde{\mathbf{u}}$, именно $\varphi^{\alpha}(k)=\tilde{u}_{k}^{\alpha}$. 
По существу, из предложения 9 следует, что координаты $(k, \tilde{\mathbf{u}})$ являются расслаивающими для пространства разностных джетов.

Пусть $\mathcal{A}(\tilde{\mathbf{V}})$ и $\mathcal{A}(\tilde{\mathbf{J}})$ - алгебры функций на пространствах $\widetilde{\mathbf{V}}$ и $\tilde{\mathbf{J}}$, соответствующие алгебрам $\mathcal{A}(\mathbf{V})$ и $\mathcal{A}(\mathbf{J})$. Фактически алгебра $\mathcal{A}(\widetilde{\mathbf{V}})$ совпадает с алгеброй $\mathcal{A}(\mathbf{V})$ (отождествление $\tilde{u}_{l}^{\alpha}=u_{l}^{\alpha}$ ), тогда как замена переменных $\varkappa: \tilde{\mathbf{J}} \simeq \mathbf{J}$ индуцирует изоморфизм

$$
\varkappa^{*}: \mathcal{A}(\mathbf{J}) \simeq \mathcal{A}(\tilde{\mathbf{J}}), \quad f \mapsto \tilde{f}=\varkappa^{*} f=f \circ \varkappa, \quad \tilde{f}(k, \tilde{\mathbf{u}})=f\left(k, T_{k} \tilde{\mathbf{u}}\right) .
$$

Группа $\mathbb{L}$ действует на алгебре $\mathcal{A}(\tilde{\mathbf{J}})$ по правилу

$$
\widetilde{T}: \mathbb{L} \rightarrow \operatorname{Aut}(\mathcal{A}(\tilde{\mathbf{J}})), \quad i \mapsto \widetilde{T}_{i}=\varkappa^{*} \circ T_{i} \circ\left(\varkappa^{*}\right)^{-1}: \mathcal{A}(\tilde{\mathbf{J}}) \rightarrow \mathcal{A}(\tilde{\mathbf{J}}),
$$

так что $\left(\widetilde{T}_{i} \tilde{f}\right)(k, \tilde{\mathbf{u}})=\tilde{f}(k+i, \tilde{\mathbf{u}})$ для всех $i, k \in \mathbb{L}, \tilde{\mathbf{u}} \in \tilde{\mathbf{J}}$ и $\tilde{f} \in \mathcal{A}(\tilde{\mathbf{J}})$.

Правило полного сдвига (предложение 2) справедливо с очевидными изменениями, алгебра $\mathcal{A}_{\widetilde{T}}(\tilde{\mathbf{J}})$ всех функций из $\mathcal{A}(\tilde{\mathbf{J}})$, инвариантных относительно сдвигов, имеет представление $\mathcal{A}_{\widetilde{T}}(\tilde{\mathbf{J}})=\mathcal{A}(\tilde{\mathbf{V}}) \subset \mathcal{A}(\tilde{\mathbf{J}})$ (см. предложение 3$)$.

$\mathcal{A}(\widetilde{\mathbf{V}})$-модуль $\mathfrak{D}(\mathcal{A}(\widetilde{\mathbf{V}}))$ имеет базис $\left\{\partial_{\tilde{u}_{l}^{\alpha}} \mid \alpha \in \mathrm{A}, l \in \mathbb{L}\right\}$, так что

$$
\mathfrak{D}(\mathcal{A}(\tilde{\mathbf{V}}))=\left\{\widetilde{X}=\sum_{\alpha, l} \tilde{X}_{l}^{\alpha} \cdot \partial_{\tilde{u}_{l}^{\alpha}} \mid \tilde{X}_{l}^{\alpha} \in \mathcal{A}(\widetilde{\mathbf{V}})\right\} .
$$

Дуальный модуль имеет дуальный базис $\left\{d_{V} \tilde{u}_{l}^{\alpha} \mid \alpha \in \mathrm{A}, l \in \mathbb{L}\right\}$, так что

$$
\mathfrak{D}^{*}(\mathcal{A}(\tilde{\mathbf{V}}))=\left\{\widetilde{\omega}=\sum_{\alpha, l} \widetilde{\omega}_{\alpha}^{l} \cdot d_{V} \tilde{u}_{l}^{\alpha} \mid \widetilde{\omega}_{\alpha}^{l} \in \mathcal{A}(\widetilde{\mathbf{V}})\right\},
$$

где лишь конечное число компонент $\widetilde{\omega}_{\alpha}^{l} \neq 0$.

Аналогичные представления имеют $\mathcal{A}(\tilde{\mathbf{J}})$-модуль $\mathfrak{D}(\mathcal{A}(\tilde{\mathbf{J}}))$ всех дифференцирований алгебры $\mathcal{A}(\tilde{\mathbf{J}})$ и дуальный $\mathcal{A}(\tilde{\mathbf{J}})$-модуль $\mathfrak{D}^{*}(\mathcal{A}(\tilde{\mathbf{J}}))$, где компоненты $\widetilde{X}_{l}^{\alpha}, \widetilde{\omega}_{\alpha}^{l} \in$ $\mathcal{A}(\tilde{\mathbf{J}})$.

Фактически алгебра Ли $\mathfrak{D}(\mathcal{A}(\tilde{\mathbf{V}}))$ совпадает с алгеброй Ли $\mathfrak{D}(\mathcal{A}(\mathbf{V}))$, а $\mathcal{A}(\tilde{\mathbf{V}})$-модуль $\mathfrak{D}^{*}(\mathcal{A}(\tilde{\mathbf{V}}))$ совпадает с $\mathcal{A}(\mathbf{V})$-модулем $\mathfrak{D}^{*}(\mathcal{A}(\mathbf{V}))$ (отождествление $\tilde{u}_{l}^{\alpha}=u_{l}^{\alpha}$, $\left.\partial_{\tilde{u}_{l}^{\alpha}}=\partial_{u_{l}^{\alpha}}, d_{V} \tilde{u}_{l}^{\alpha}=d_{V} u_{l}^{\alpha}\right)$, тогда как замена переменных $\varkappa: \tilde{\mathbf{J}} \simeq \mathbf{J}$ индуцирует изоморфизмы

$$
\varkappa_{*}: \mathfrak{D}(\mathcal{A}(\tilde{\mathbf{J}})) \simeq \mathfrak{D}(\mathcal{A}(\mathbf{J})), \quad \varkappa^{*}: \mathfrak{D}^{*}(\mathcal{A}(\mathbf{J})) \simeq \mathfrak{D}^{*}(\mathcal{A}(\tilde{\mathbf{J}}))
$$

согласно правилам

$$
\widetilde{X} \mapsto X=\varkappa_{*} \widetilde{X}=\left(\varkappa^{*}\right)^{-1} \circ \widetilde{X} \circ \varkappa^{*}, \quad \omega \mapsto \widetilde{\omega}=\varkappa^{*} \omega=\varkappa^{*} \circ \omega \circ \varkappa_{*} .
$$

В частности,

1) $\partial_{\tilde{u}_{l}^{\alpha}} \mapsto \partial_{\alpha}^{l}=\partial_{u_{l-k}^{\alpha}}, \partial_{u_{l}^{\alpha}} \mapsto \widetilde{\partial}_{\alpha}^{l}=\partial_{\tilde{u}_{l+k}^{\alpha}}$;

2) $\tilde{X}=\sum_{\alpha, l} \tilde{X}_{l}^{\alpha} \cdot \partial_{\tilde{u}_{l}^{\alpha}} \mapsto X=\sum_{\alpha, l} X_{l}^{\alpha} \cdot \partial_{u_{l}^{\alpha}}, \quad X_{l}^{\alpha}(k, \mathbf{u})=\tilde{X}_{l+k}^{\alpha}\left(k, T_{-k} \mathbf{u}\right)$;

3) $d_{V} u_{l}^{\alpha} \mapsto \widetilde{\vartheta}_{l}^{\alpha}=d_{V} \tilde{u}_{l+k}^{\alpha}, \quad d_{V} \tilde{u}_{l}^{\alpha} \mapsto \vartheta_{l}^{\alpha}=d_{V} u_{l-k}^{\alpha}$; 
4) $\omega=\sum_{\alpha, l} \omega_{\alpha}^{l} \cdot d_{V} u_{l}^{\alpha} \mapsto \widetilde{\omega}=\sum_{\alpha, l} \widetilde{\omega}_{\alpha}^{l} \cdot d_{V} \tilde{u}_{l}^{\alpha}, \quad \widetilde{\omega}_{\alpha}^{l}(k, \tilde{\mathbf{u}})=\omega_{\alpha}^{l-k}\left(k, T_{k} \tilde{\mathbf{u}}\right)$;

5) $\langle\widetilde{\omega}, \widetilde{X}\rangle=\varkappa^{*}\langle\omega, X\rangle$ для всех $\omega \in \mathfrak{D}^{*}(\mathcal{A}(\mathbf{J})), X \in \mathfrak{D}(\mathcal{A}(\mathbf{J}))$.

Поясним, что, например, $\left(\partial_{\alpha}^{l} f\right)(k, \mathbf{u})=\partial_{u_{l-k}^{\alpha}} f(k, \mathbf{u}), f \in \mathcal{A}(\mathbf{J})$.

Группа $\mathbb{L}$ действует на $\mathcal{A}(\tilde{\mathbf{J}})$-модулях $\mathfrak{D}(\mathcal{A}(\tilde{\mathbf{J}}))$ и $\mathfrak{D}^{*}(\mathcal{A}(\tilde{\mathbf{J}}))$ :

$$
\widetilde{T}: \mathbb{L} \rightarrow \operatorname{Aut}(\mathfrak{D}(\mathcal{A}(\tilde{\mathbf{J}}))), \quad i \mapsto \widetilde{T}_{i}, \quad \widetilde{T}: \mathbb{L} \rightarrow \operatorname{Aut}\left(\mathfrak{D}^{*}(\mathcal{A}(\tilde{\mathbf{J}}))\right), \quad i \mapsto \widetilde{T}_{i},
$$

согласно правилам

$$
\widetilde{X} \mapsto \widetilde{T}_{i} \widetilde{X}=\widetilde{T}_{i} \circ \tilde{X}_{\circ} \circ \widetilde{T}_{-i}, \quad \widetilde{\omega} \mapsto \widetilde{T}_{i} \widetilde{\omega}=\widetilde{T}_{i} \circ \widetilde{\omega} \circ \widetilde{T}_{-i}
$$

или подробнее

$$
\begin{array}{ll}
\widetilde{X}=\sum_{\alpha, l} \widetilde{X}_{l}^{\alpha} \cdot \partial_{\tilde{u}_{l}^{\alpha}} \mapsto \widetilde{T}_{i} \widetilde{X}=\sum_{\alpha, l} \widetilde{T}_{i} \widetilde{X}_{l}^{\alpha} \cdot \partial_{\tilde{u}_{l}^{\alpha}}, & \widetilde{T}_{i} \widetilde{X}_{l}^{\alpha}(k, \tilde{\mathbf{u}})=\widetilde{X}_{l}^{\alpha}(k+i, \tilde{\mathbf{u}}), \\
\widetilde{\omega}=\sum_{\alpha, l} \widetilde{\omega}_{\alpha}^{l} \cdot d_{V} \tilde{u}_{l}^{\alpha} \mapsto \widetilde{T}_{i} \widetilde{\omega}=\sum_{\alpha, l} \widetilde{T}_{i} \widetilde{\omega}_{\alpha}^{l} \cdot d_{V} \widetilde{\tilde{u}}_{l}^{\alpha}, & \widetilde{T} \widetilde{\omega}_{\alpha}^{l}(k, \tilde{\mathbf{u}})=\widetilde{\omega}_{\alpha}^{l}(k+i, \tilde{\mathbf{u}}) .
\end{array}
$$

В частности, справедливы следующие утверждения:

1) базис $\left\{\partial_{\tilde{u}_{l}^{\alpha}} \mid \alpha \in \mathrm{A}, l \in \mathbb{L}\right\}$ в $\mathcal{A}(\tilde{\mathbf{J}})$-модуле $\mathfrak{D}(\mathcal{A}(\tilde{\mathbf{J}}))$ инвариантен относительно сдвигов, т. е. $\widetilde{T}_{i} \partial_{\tilde{u}_{l}^{\alpha}}=\partial_{\tilde{u}_{l}^{\alpha}}$ для всех $\alpha \in \mathrm{A}, l \in \mathbb{L}$;

2) множество $\left\{\partial_{\alpha}^{l} \mid \alpha \in \mathrm{A}, l \in \mathbb{L}\right\}$ есть базис в $\mathcal{A}(\mathbf{J})$-модуле $\mathfrak{D}(\mathcal{A}(\mathbf{J}))$, инвариантный относительно сдвигов, т. е. $T_{i} \partial_{l}^{\alpha}=\partial_{l}^{\alpha}$ для всех $\alpha \in \mathrm{A}, l \in \mathbb{L}$;

3) базис $\left\{d_{V} \tilde{u}_{l}^{\alpha} \mid \alpha \in \mathrm{A}, l \in \mathbb{L}\right\}$ в $\mathcal{A}(\tilde{\mathbf{J}})$-модуле $\mathfrak{D}^{*}(\mathcal{A}(\tilde{\mathbf{J}}))$ инвариантен относительно сдвигов, т. е. $\widetilde{T}_{i} d_{V} \tilde{u}_{l}^{\alpha}=d_{V} \tilde{u}_{l}^{\alpha}$ для всех $\alpha \in \mathrm{A}, l \in \mathbb{L}$;

4) множество $\left\{\vartheta_{l}^{\alpha} \mid \alpha \in \mathrm{A}, l \in \mathbb{L}\right\}$ есть базис в $\mathcal{A}(\mathbf{J})$-модуле $\mathfrak{D}^{*}(\mathcal{A}(\mathbf{J}))$, инвариантный относительно сдвигов, т. е. $T_{i} \vartheta_{l}^{\alpha}=\vartheta_{l}^{\alpha}$ для всех $\alpha \in \mathrm{A}, l \in \mathbb{L}$;

5) алгебра Ли дифференцирований, инвариантных относительно сдвигов (см. предложение 4), алгебры $\mathcal{A}(\tilde{\mathbf{J}})$ имеет представление

$$
\mathfrak{D}_{T}(\mathcal{A}(\tilde{\mathbf{J}}))=\left\{\tilde{X}=\sum_{\alpha, l} \tilde{f}_{l}^{\alpha} \cdot \partial_{\tilde{u}_{l}^{\alpha}} \mid \tilde{f}_{l}^{\alpha} \in \mathcal{A}(\tilde{\mathbf{V}})\right\}=\mathfrak{D}(\mathcal{A}(\tilde{\mathbf{V}})) .
$$

Определена внешняя алгебра $\Omega(\widetilde{\mathbf{V}})$, совпадающая с алгеброй $\Omega(\mathbf{V})$, причем однозначно определен вертикальный дифференциал $\widetilde{d}_{V}: \Omega(\widetilde{\mathbf{V}}) \rightarrow \Omega(\widetilde{\mathbf{V}})$, совпадающий с вертикальным дифференциалом $d_{V}: \Omega(\mathbf{V}) \rightarrow \Omega(\mathbf{V})$, так что комплекс $\left\{\Omega(\widetilde{\mathbf{V}}) ; \widetilde{d}_{V}\right\}$ совпадает с комплексом $\left\{\Omega(\mathbf{V}) ; d_{V}\right\}$, и в силу теоремы 1 пополненный комплекс

$$
0 \longrightarrow \mathbb{R} \longrightarrow \Omega^{0}(\tilde{\mathbf{V}}) \stackrel{\widetilde{d}_{V}}{\longrightarrow} \Omega^{1}(\tilde{\mathbf{V}}) \stackrel{\widetilde{d}_{V}}{\longrightarrow} \Omega^{2}(\tilde{\mathbf{V}}) \stackrel{\widetilde{d}_{V}}{\longrightarrow} \Omega^{3}(\tilde{\mathbf{V}}) \stackrel{\widetilde{d}_{V}}{\longrightarrow} \cdots
$$

точный, т. е. все его пространства когомологий тривиальные.

Разностные операторы $\widetilde{\Delta}_{\mu}=\widetilde{T}_{e_{\mu}}-\widetilde{T}_{0}, \mu \in \mathbb{D}$, действуют во всех пространствах, где определено действие группы сдвигов $\mathbb{L}$, причем $\widetilde{\Delta}_{\mu}=\Delta_{\mu}$ на алгебре $\mathcal{A}(\mathbb{L})$, но $\widetilde{\Delta}_{\mu} \neq$ $\Delta_{\mu}$ на алгебрах $\mathcal{A}(\tilde{\mathbf{V}})$ и $\mathcal{A}(\tilde{\mathbf{J}})$. В частности, $\mathcal{A}(\mathbb{L})$-модули $\mathfrak{D}(\mathbb{L})$ и $\mathfrak{D}(\mathbb{L})$ сохраняют свой вид и действие $\widetilde{T}=T$ на них. 
По-прежнему определены градуированная внешняя алгебра $\Omega(\mathbb{L})$, горизонтальный дифференциал $d_{H}: \Omega(\mathbb{L}) \rightarrow \Omega(\mathbb{L})$ и горизонтальный комплекс $\left\{\Omega(\mathbb{L}) ; d_{H}\right\}$, причем теорема 2 остается в силе.

Биградуированная внешняя алгебра дифференциально-разностных форм на пространстве $\tilde{\mathbf{J}}$ имеет вид

$$
\Omega(\tilde{\mathbf{J}})=\bigoplus_{p \in \mathbb{Z}_{+}, 0 \leqslant q \leqslant D} \Omega^{p q}(\tilde{\mathbf{J}}), \quad \Omega^{p q}(\tilde{\mathbf{J}})=\Omega^{p}(\tilde{\mathbf{V}}) \wedge_{\mathbb{R}} \Omega^{q}(\mathbb{L}) .
$$

По построению имеем изоморфизм биградуированных внешних алгебр

$$
\varkappa^{*}: \Omega(\mathbf{J}) \simeq \Omega(\tilde{\mathbf{J}})
$$

определяемый правилами: $f \mapsto \tilde{f}, d_{V} u_{l}^{\alpha} \mapsto \widetilde{\vartheta}_{l}^{\alpha}, d_{H} i^{\mu} \mapsto d_{H} i^{\mu}$. Более того, однозначно определены вертикальный и горизонтальный дифференциалы $\widetilde{d}_{V}$ и $\widetilde{d}_{H}$ такие, что имеет место изоморфизм бикомплексов

$$
\varkappa^{*}:\left\{\Omega(\mathbf{J}) ; d_{V}, d_{H}\right\} \simeq\left\{\Omega(\tilde{\mathbf{J}}) ; \tilde{d}_{V}, \tilde{d}_{H}\right\} .
$$

Именно, по-прежнему вертикальный дифференциал

$$
\widetilde{d}_{V}=\widetilde{d}_{V}^{p q}=\widetilde{d}_{V}^{p} \wedge \operatorname{id}_{\Omega^{q}(\mathbb{L})}: \Omega^{p q}(\tilde{\mathbf{J}}) \rightarrow \Omega^{p+1, q}(\tilde{\mathbf{J}})
$$

так что $\widetilde{d}_{V}(\chi \wedge \varrho)=\left(\widetilde{d}_{V} \chi\right) \wedge \varrho$ для всех $\chi \in \Omega^{p}(\widetilde{\mathbf{V}}), \varrho \in \Omega^{q}(\mathbb{L})$. Но теперь и горизонтальный дифференциал факторизуется:

$$
\widetilde{d}_{H}=\widetilde{d}_{H}^{p q}=\operatorname{id}_{\Omega^{p}(\tilde{\mathbf{V}})} \wedge d_{H}^{q}: \Omega^{p q}(\tilde{\mathbf{J}}) \rightarrow \Omega^{p, q+1}(\tilde{\mathbf{J}}),
$$

так что $\widetilde{d}_{H}(\chi \wedge \varrho)=(-1)^{p} \chi \wedge d_{H}(\varrho)$ для всех $\chi \in \Omega^{p}(\tilde{\mathbf{V}}), \varrho \in \Omega^{q}(\mathbb{L})$. Именно с этой целью и были введены расслаивающие координаты.

ЗАмЕчАниЕ 2. В случае вариационного исчисления на евклидовом пространстве (см., например, [3], [4]) такие координаты отсутствуют, чем и объясняется существенная разница свойств вариационного бикомплекса и дифференциально-разностного бикомплекса.

В свою очередь, действие $\widetilde{T}: \mathbb{L} \rightarrow \operatorname{Aut}(\Omega(\tilde{\mathbf{J}}))$ также факторизуется. Именно, $\widetilde{T}_{i}(\chi \wedge \varrho)=\chi \wedge \widetilde{T}_{i} \varrho$ для всех $\chi \in \Omega(\widetilde{\mathbf{V}})$ и $\varrho \in \Omega(\mathbb{L})$. Разностная форма $\varrho=$ $\frac{1}{q !} \sum_{|\boldsymbol{\mu}|=q} \varrho_{\boldsymbol{\mu}} \cdot d_{H} i^{\boldsymbol{\mu}}$ инвариантна относительно сдвигов тогда и только тогда, когда $\varrho_{\boldsymbol{\mu}} \in \mathbb{R} \subset \mathcal{A}(\mathbb{L})$. Следовательно, $\mathcal{A}(\widetilde{\mathbf{V}})$-модули инвариантных форм суть

$$
\Omega_{\widetilde{T}}^{p q}(\tilde{\mathbf{J}})=\left\{\omega=\frac{1}{q !} \sum_{|\boldsymbol{\mu}|=q} \chi_{\boldsymbol{\mu}} \wedge d_{H} i^{\boldsymbol{\mu}} \mid \chi_{\boldsymbol{\mu}} \in \Omega^{p}(\tilde{\mathbf{V}})\right\}
$$

Изоморфизм $\varkappa^{*}: \Omega_{T}(\mathbf{J}) \simeq \Omega_{\widetilde{T}}(\tilde{\mathbf{J}})$ позволяет дать явное описание $\mathcal{A}_{T}(\mathbf{J})$-модулей $\Omega_{T}^{p q}(\mathbf{J})$. Именно, справедливо следующее предложение. 


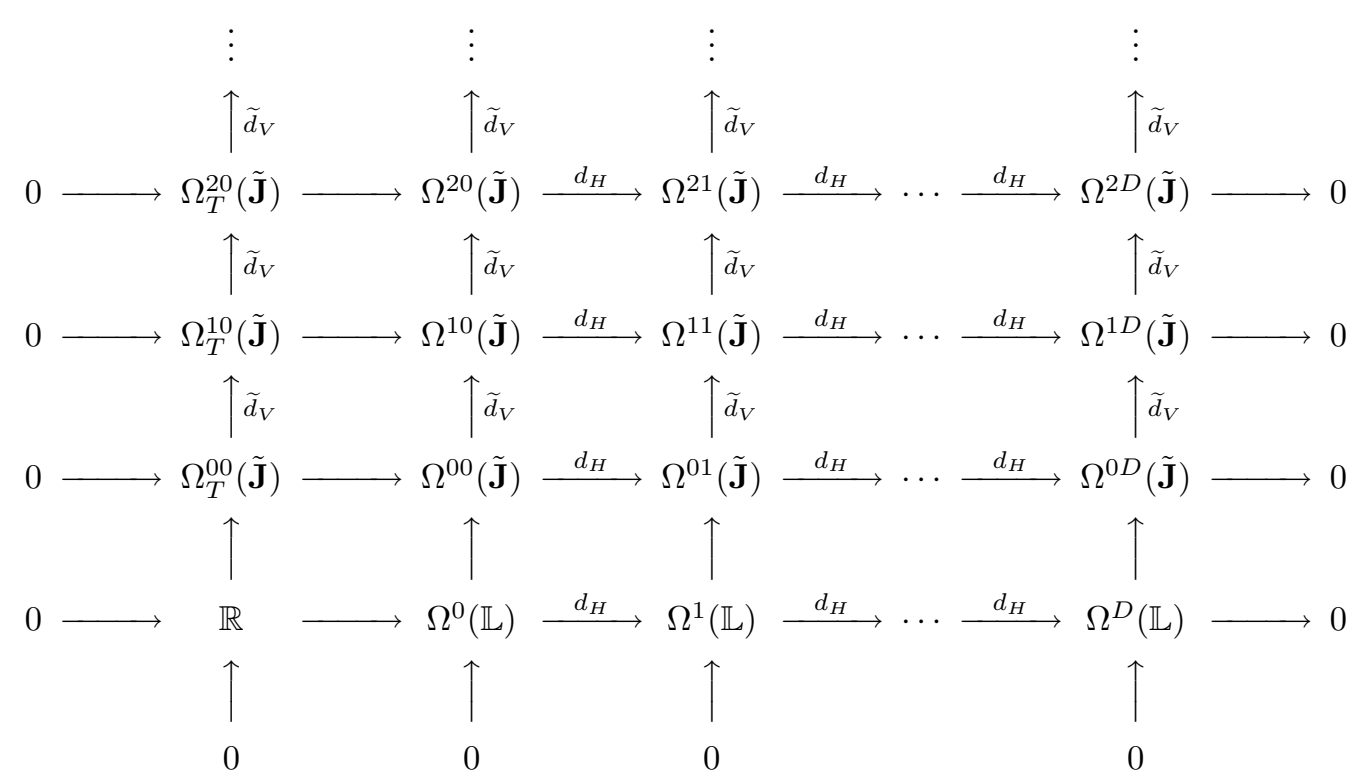

Рис. 2. Пополненный бикомплекс на пространстве $\tilde{\mathbf{J}}$.

ПреДЛОЖениЕ 10. Для всех $p \in \mathbb{Z}_{+}, 0 \leqslant q \leqslant D$ имеет место представление

$$
\Omega_{T}^{p q}(\mathbf{J})=\left\{\omega=\frac{1}{q !} \sum_{|\boldsymbol{\mu}|=q} \chi_{\boldsymbol{\mu}} \wedge d_{H} i^{\boldsymbol{\mu}} \mid \chi_{\boldsymbol{\mu}} \in \Omega_{T}^{p 0}(\mathbf{J})\right\}
$$

где

$$
\Omega_{T}^{p 0}(\mathbf{J})=\left\{\frac{1}{p !} \sum_{|\boldsymbol{\alpha}, \mathbf{i}|=p} \chi_{\boldsymbol{\alpha}}^{\mathbf{i}} \cdot \vartheta_{\mathbf{i}}^{\boldsymbol{\alpha}} \mid \chi_{\boldsymbol{\alpha}}^{\mathbf{i}} \in \mathcal{A}_{T}(\mathbf{J})\right\} .
$$

Из формулы $\widetilde{d}_{V}=\sum_{\alpha, i} \widetilde{\varepsilon}_{i}^{\alpha} \circ \partial_{\mathbf{u}_{i}^{\alpha}}$ следует, что определено сужение вертикального дифференциала на инвариантные формы,

$$
\widetilde{d}_{V}: \Omega_{\widetilde{T}}^{p q}(\tilde{\mathbf{J}}) \rightarrow \Omega_{\widetilde{T}}^{p+1, q}(\tilde{\mathbf{J}}) \quad \text { для всех } \quad p \in \mathbb{Z}_{+}, 0 \leqslant q \leqslant D .
$$

Итак, определен пополненный бикомплекс, изображенный на рис. 2, изоморфный бикомплексу, изображенному на рис. 1.

Теорема 4. Пополненный бикомплекс, изображенный на рис. 2, ациклический, m.е. все его строки и столбць точные.

ДокАЗАТЕЛьство. Действительно, все строки рассматриваемого бикомплекса точные в силу теоремы 2 , поскольку горизонтальный дифференциал факторизован. Аналогично все столбцы бикомплекса, кроме, быть может, крайнего левого, точные в силу теоремы 1 , поскольку вертикальный дифференциал тоже факторизован. Для 
доказательства точности крайнего левого столбца следует выписать явные формулы для гомотопического оператора $h_{V}$ (см. доказательство теоремы 1$)$ и убедиться, что определено его сужение на инвариантные формы.

СледСтвиЕ 2. Справедлива теорема 3.

Для доказательства достаточно воспользоваться изоморфизмом пополненных бикомплексов.

2.5. Обсуждение. Основные вопросы, возникающие при осмыслении теоремы 3: где разностные функциональные формы и вариационные производные и где разностное вариационное исчисление? Ответ простой: сделанный здесь выбор в качестве класса функций на пространстве разностных джетов алгебры $\mathcal{A}(\mathbf{J})$, а не ее подалгебры $\mathcal{A}_{\text {fin }}(\mathbf{J})$ допускает лишь тривиальные нулевые функциональные формы и как следствие вырождение вариационного исчисления. Поясним сказанное на примерах (для простоты полагаем $\mathbb{L}=\mathbb{Z}$ и $V=\mathbb{R}$ ).

Пример 1. В алгебре $\mathcal{A}(\mathbf{J})$ каждая функция имеет разностную первообразную. Действительно, для данной функции $f(k, \mathbf{u}) \in \mathcal{A}(\mathbf{J})$ положим

$$
F(k, \mathbf{u})= \begin{cases}\sum_{0 \leqslant i<k} f\left(i, T_{i-k} \mathbf{u}\right), & k>0, \\ 0, & k=0, \\ -\sum_{k \leqslant i<0} f\left(i, T_{i-k} \mathbf{u}\right), & k<0 .\end{cases}
$$

Тогда $F(k, \mathbf{u}) \in \mathcal{A}(\mathbf{J})$ и $\Delta F=f$ (точнее, $\left.F\left(k+1, T_{1} \mathbf{u}\right)-F(k, \mathbf{u})=f(k, \mathbf{u})\right)$. Заметим, что построенная первообразная $F \notin \mathcal{A}_{\mathrm{fin}}(\mathbf{J})$.

Пример 2. Разностная вариационная производная $\delta f / \delta u$ функции $f(k, \mathbf{u})$ определяется формулой (см., например, [7], [8])

$$
\frac{\delta f}{\delta u}(k, \mathbf{u})=\sum_{i \in \mathbb{L}} T_{-i} \frac{\partial}{\partial u_{i}} f(k, \mathbf{u})=\sum_{i \in \mathbb{L}} \frac{\partial}{\partial u_{0}} T_{-i} f(k, \mathbf{u}) .
$$

По построению сумма по $i$ конечная, если $f \in \mathcal{A}_{\mathrm{fin}}(\mathbf{J})$, так что разностная вариационная производная определена на алгебре $\mathcal{A}_{\mathrm{fin}}(\mathbf{J})$. С другой стороны, для некоторых $f \in \mathcal{A}(\mathbf{J})$ эта сумма может оказаться бесконечной. Например, пусть $f \in \mathcal{A}_{T}(\mathbf{J})$, так что $T_{i} f=f$ для всех $i \in \mathbb{L}$, тогда

$$
\frac{\delta f}{\delta u}(k, \mathbf{u})=\sum_{i \in \mathbb{L}} \frac{\partial f(k, \mathbf{u})}{\partial u_{0}}=\left\{\begin{array}{lll}
\infty, & \text { если } & \frac{\partial f(k, \mathbf{u})}{\partial u_{0}} \neq 0 \\
0, & \text { если } & \frac{\partial f(k, \mathbf{u})}{\partial u_{0}}=0
\end{array}\right.
$$

т. е. разностная вариационная производная не определена для любой функции из $\mathcal{A}_{T}(\mathbf{J})$. 
Таким образом, для развития разностного вариационного исчисления алгебра $\mathcal{A}(\mathbf{J})$ не подходит, более перспективной выглядит ее подалгебра $\mathcal{A}_{\text {fin }}(\mathbf{J})$. Отметим, что в работах на эту тему (см., например, [7]-[14]) негласно используются алгебра $\mathcal{A}_{\text {fin }}(\mathbf{J})$ и ее подалгебра $\mathcal{A}(\mathbf{V})$. Также отметим, что ситуация с разностным вариационным комплексом, а тем более с бикомплексом, далеко не ясна, несмотря на общее мнение о схожести разностного вариационного комплекса на целочисленной решетке с вариационным комплексом на евклидовом пространстве. Например, в работе [11] объявлено о доказательстве точности (ацикличности) разностного вариационного комплекса, однако доказательство главной теоремы 4.2.31 отсутствует, а построенный в ней гомотопический оператор является калькой аналогичного оператора для вариационного комплекса на евклидовом пространстве (см., например, [1]) и не отражает принципиальной разницы между разностными операторами на целочисленной решетке и полными производными на евклидовом пространстве (ср. с замечанием 1). Во всяком случае задача построения разностного вариационного бикомплекса к классе $\mathcal{A}_{\text {fin }}(\mathbf{J})$ и доказательства его ацикличности еще впереди.

Другой важный вопрос, какой класс функций лучше подходит для приложений. Здесь также ответ простой: как и в евклидовом случае, где нет лучшего класса, для различных задач математической физики используется широкий спектр функциональных пространств, выбор класса функций на пространстве разностных джетов диктуется конкретной поставленной задачей. Например, в конкретной прикладной задаче вполне может возникнуть разностное уравнение вида $F(k, \mathbf{u})=0$, где функция $F \in \mathcal{A}(\mathbf{J}) \backslash \mathcal{A}_{\text {fin }}(\mathbf{J})$, так что при исследовании такого уравнения придется работать в алгебре $\mathcal{A}(\mathbf{J})$. С другой стороны, согласно современным воззрениям каждая фундаментальная модель, в частности на решетке, должна быть лагранжевой, т. е. задаваться вариационными уравнениями $\delta L / \delta u^{\alpha}=0, \alpha \in \mathrm{A}$, где лагранжиан $L=L(\mathbf{u}) \in \mathcal{A}(\mathbf{V}) \subset \mathcal{A}_{\text {fin }}(\mathbf{J})$, так что в этом случае достаточно алгебры $\mathcal{A}_{\text {fin }}(\mathbf{J})$ или ее подалгебры $\mathcal{A}(\mathbf{V})$.

Благодарности. Работа выполнена при поддержке РФФИ (гранты № 10-0100178, 09-026-12161-офи-м) и Программы поддержки ведущих научных школ (грант НШ-7675.2010.1).

\section{Список литературы}

[1] П. Олвер, Приложения групп Ли к дифференииальным уравнениям, Мир, М., 1989.

[2] T. Tsujishita, Osaka J. Math., 19:2 (1982), 311-363.

[3] I. M. Anderson, "Introduction to the variational bicomplex", Mathematical Aspects of Classical Field Theory, Contemp. Math., 132, eds. M. J. Gotay, J. E. Marsden, V. Moncrief, AMS, Providence, RI, 1992, 51-73.

[4] I. M. Anderson, The Variational Bicomplex, Dept. Math., Utah State University, Logan, Utah, 2004, 318 pp.

[5] А. Картан, Дифференииалъное исчисление. Дифференииалъные формы, Мир, М., 1971.

[6] В. В. ЖКаринов, ТМФ, 144:3 (2005), 435-452.

[7] B. Kupershmidt, Discrete Lax equations and differential-difference calculus, Astérisque, 123, SMF, Paris, 1985.

[8] P. E. Hydon, E. L. Mansfield, Found. Comput. Math., 4:2 (2004), 187-217.

[9] Ü. Göktaş, W. Hereman, Physica D, 123:1-4 (1998), 425-436, arXiv: solv-int/9801023. 
[10] В. Э. Адлер, А. Б. Шабат, Р. И. Ямилов, ТМФ, 125:3 (2000), 355-424.

[11] E. L. Mansfield, P. E. Hydon, "On a variational complex for difference equations", The Geometrical Study of Differential Equations, Contemp. Math., 285, eds. J. A. Leslie, T. P. Robart, AMS, Providence, RI, 2002, 121-129.

[12] M. S. Hickman, W. A. Hereman, Proc. Roy. Soc. A, 459 (2003), 2705-2729.

[13] W. Hereman, M. Colagrosso, R. Sayers, A. Ringler, B. Deconinck, M. Nivala, M. Hickman, "Continuous and discrete homotopy operators and the computation of conservation laws", Differential Equations With Symbolic Computation, Trends Math., eds. D. Wang, Z. Zheng, Birkhäuser, Basel, 2005, 255-290.

[14] В. В. Жаринов, ТМФ, 157:3 (2008), 391-405.

Поступила в редакцию 10.08.2010 